\title{
ESTRATEGIAS HUMANAS, ESTABILIDAD Y CAMBIO EN LA FRONTERA AGRÍCOLA SUR AMERICANA
}

\author{
Adolfo F. Gil, Lumila P. Menéndez, Juan P. Atencio, Eva A. Peralta, Gustavo A. Neme \\ y Andrew Ugan
}

\begin{abstract}
El trabajo explora cuán estables fueron las estrategias humanas una vez que las poblaciones indígenas del centro-occidente argentino incorporaron cultígenos. Asimismo, evalúa si una vez incorporado el consumo de plantas domésticas, las estrategias fueron, o no, progresivamente apoyándose más sobre la producción y consumo de las mismas. El estudio compara tres sitios con restos humanos (Jaime Prats-1, Rincón del Atuel-1 y Cañada Seca-1) localizados entre sí a una distancia máxima de $40 \mathrm{~km}$ y en un valle considerado como el límite sur de la agricultura andina prehispánica. Estos sitios, que presentan una continuidad temporal, abarcan desde ca. 1900 (300 años posterior al registro inicial de las plantas domésticas en la región) a ca. 1400 años aP. Se comparan datos de isótopos estables $\left({ }^{13} \mathrm{C},{ }^{15} \mathrm{~N},{ }^{18} \mathrm{O}\right)$, morfometría craneal, mandibular y dental, junto a información contextual arqueológica. El sitio más antiguo, Jaime Prats-1, muestra semejanzas isotópicas y morfológicas con Cañada Seca-1, el más reciente, a pesar de no ser continuos en el tiempo. Ambos se diferencian de Rincón del Atuel-1, que temporalmente se intercala entre los dos. Los resultados señalan una mayor importancia del maíz en Rincón del Atuel-1, junto a una menor movilidad residencial, aunque en ninguno de los casos este recurso habría contribuido más del $30 \%$ a la dieta. Los sistemas humanos que ocuparon la frontera agrícola en estos primeros 500 años de uso de plantas domésticas muestran una alta variabilidad en su organización, sin cruzar umbrales que impidan retornar a sistemas de baja producción.
\end{abstract}

In this paper, we assess the impact of the introduction of cultigens on human organization and the resultant degree of dependence on food production and domesticated plants in central western Argentina. The study compares three sites with human skeletal remains (Jaime Prats-1, Rincón del Atuel-1, Cañada Seca-1) separated by a maximum distance of $40 \mathrm{~km}$ and located in the southernmost region of Andean prehispanic agriculture. The sites have regional temporal occupation continuity dating back to 1900 (300 years after introduction of domesticated plants) to 1400 years BP. Data from stable isotopes $\left({ }^{13} \mathrm{C},{ }^{15} \mathrm{~N}\right.$, $\left.{ }^{18} \mathrm{O}\right)$, and cranial, mandibular, and dental morphometry are compared, along with archaeological information. Jaime Prats-1 and Cañada Seca-1 exhibit isotopic and morphologic similarities, despite not being chronologically contemporaneous. At the same time, these two sites have differences with Rincón del Atuel-1, temporally placed among them. Our results point to a greater importance of maize (although its dietary contribution is less than 30\%) and lower residential mobility in Rincón del Atuel-1. Human systems that occupied the agricultural frontier during the first 500 years since the introduction of domesticated plants show high variability in their organizations, with agriculture playing a minor role in sustenance.

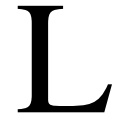
a incorporación de plantas domésticas entre poblaciones humanas ha sido clásicamente asociada a la adopción de la agricultura y a la disminución de la movilidad
(Cohen 1977). Estudios recientes muestran que la transición no necesariamente fue abrupta, lineal e irreversible. También señalan que el registro de plantas domésticas no implica agricultura

\footnotetext{
Adolfo F. Gil — CONICET/IANIGLA-Grupo Vinculado San Rafael, San Rafael, Mendoza, Argentina, CP 5600

(agil@mendoza-conicet.gob.ar, autor de contacto)

Lumila P. Menéndez $\varpi$ DFG Center for Advanced Studies, University of Tübingen, Rümelinstraße 23, Tübingen, Germany, 72070.

Juan P. Atencio — CONICET, División Antropología, Museo de Ciencias Naturales, Facultad de Ciencias Naturales y Museo de La Plata, Universidad Nacional de La Plata, La Plata, Buenos Aires, Argentina, CP 1900.

Eva A. Peralta $\square$ CONICET/IANIGLA-Grupo Vinculado San Rafael, San Rafael, Mendoza, Argentina, CP 5600. Gustavo A. Neme CONICET/IANIGLA-Grupo Vinculado San Rafael, San Rafael, Mendoza, Argentina, CP 5600. Andrew Ugan $\square$ Far Western Anthropological Research Group, Davis, California 95618 USA \& Museo de Historia Natural de San Rafael, San Rafael, Mendoza, Argentina, CP 5600.
}

Latin American Antiquity, page 1 of 21

Copyright (C) 2017 by the Society for American Archaeology doi:10.1017/laq.2017.59 
$\mathrm{y}$ que hay un rango de estrategias diversas entre los extremos de cazadores-recolectores y productores (Smith 2001). Estas propuestas surgieron en áreas vinculadas al origen de la domesticación, y enfocadas en las causas de la misma. Sin embargo, se ha puesto poco esfuerzo en entender la forma y causa de su dispersión. El registro arqueológico del centro-occidente argentino permite profundizar el entendimiento de este proceso asociado a la introducción de la producción de alimentos. Esta región es presentada como el límite meridional andino en el registro de cultígenos prehispánicos en América (Gil 1998; Gil et al. 2014a; Lagiglia 1982). Específicamente, el valle del Río Atuel (Figura 1) fue propuesto como el lugar hasta el cual llegaron los primeros asentamientos de agricultores alrededor de 2200 años aP (Lagiglia 1982). La cultura Atuel II habría introducido la agricultura y como tal se habría asentado en el mencionado valle hasta hace unos 1800 años aP (Lagiglia 1982). Otras investigaciones señalaron que los cultígenos en ese momento no fueron elementos importantes en la dieta humana, aunque podrían haber tenido un valor simbólico (Gil 2003). El primer modelo arqueológico (Lagiglia 1982) sobre el desarrollo cultural de este valle no explicitó qué estrategias de subsistencia se sucedieron entre Atuel II (2200 a 1800 años aP) y Atuel I (300 años aP) dejando de ese modo unos 1.500 años sin asignación explícita a contextos arqueológicos, ni explicaciones de los procesos ocurridos durante ese tiempo. Por eso, en base a los hallazgos, se asumió para ese lapso la presencia de ocupaciones agroalfareras de tipo Agrelo y Viluco provenientes del norte de Mendoza (Lagiglia 2002). La situación e importancia posterior a esta fase inicial de introducción de cultígenos no ha sido abordada explícitamente y aunque se asumió un incremento de los mismos en la dieta, para épocas históricas se han descripto ocupaciones de cazadores-recolectores sin mencionarse el consumo de plantas domésticas. Cuán significativos fueron los recursos vegetales domésticos en la dieta humana, y cuán estables fueron las estrategias y organización en esta zona de frontera, son los temas centrales abordados en este trabajo. Aquí se indaga el tema usando principalmente información bioarqueológica. Se seleccionaron tres sitos con entierros humanos
(Jaime Prats-1, Rincón del Atuel-1 y Cañada Seca-1; Figura 1), próximos tanto en el espacio (todos en distancia inferiores a $40 \mathrm{~km}$ ), así como en el tiempo (entre ca. 2000 y 1500 años aP). Se presenta un análisis de la variación isotópica de ${ }^{13} \mathrm{C},{ }^{15} \mathrm{~N}$ y ${ }^{18} \mathrm{O}$ para discutir dieta y movilidad, así como también de la variación morfológica craneofacial y dental. Finalmente, se evalúa la asociación entre la información isotópica y morfológica mediante regresiones espaciales.

\section{Cultígenos y Estrategias Humanas en los Límites Prehispánicos de la Producción}

El centro-occidente argentino, definido como una unidad espacial heurística, es considerado, junto a Chile central, el límite meridional de la agricultura andina americana. Específicamente, en el sector oriental los ríos Diamante y Atuel (aproximadamente $34^{\circ} 30^{\prime}$ S; Figura 1) habrían funcionado durante los últimos 2.000 años como la frontera sur de la agricultura prehispánica (Gil y Neme 2013; Gil et al. 2014b). Hacia el sur de dichos ríos la caza y recolección continuó como forma de vida, incluso hasta el siglo XIX (Gil y Neme 2013).

Lagiglia (1982) planteó que los primeros agricultores se establecieron en este valle sobre la base de grupos de familias que arribaron trayendo el conocimiento y los elementos para esta nueva forma de vida. Posteriormente, denominó a ese proceso como "exploradores y colonizadores agrícolas iniciales". Estas poblaciones fueron caracterizadas como de baja densidad demográfica (Lagiglia 2001). Para Lagiglia (2001) el posterior desarrollo aldeano y la incorporación de la tecnología cerámica se asociarían a incrementos demográficos de estos grupos.

Posteriores investigaciones en la región postularon el inicio de importantes cambios en la organización humana en torno a los 2000 años aP (Gil et al. 2014a; Lagiglia 1982; Neme 2007; Neme y Gil 2008). Gil y colaboradores (2014a) señalaron que los primeros cultígenos de la región, según fechas radiocarbónicas directas, se registran entre 2200 y 2050 años ${ }^{14} \mathrm{C}$ aP, en la Gruta del Indio. Esos primeros cultígenos no habrían tenido una contribución importante en la dieta, sino más bien habrían portado un rol simbólico (Gil 2003). Esto se sumó a la 


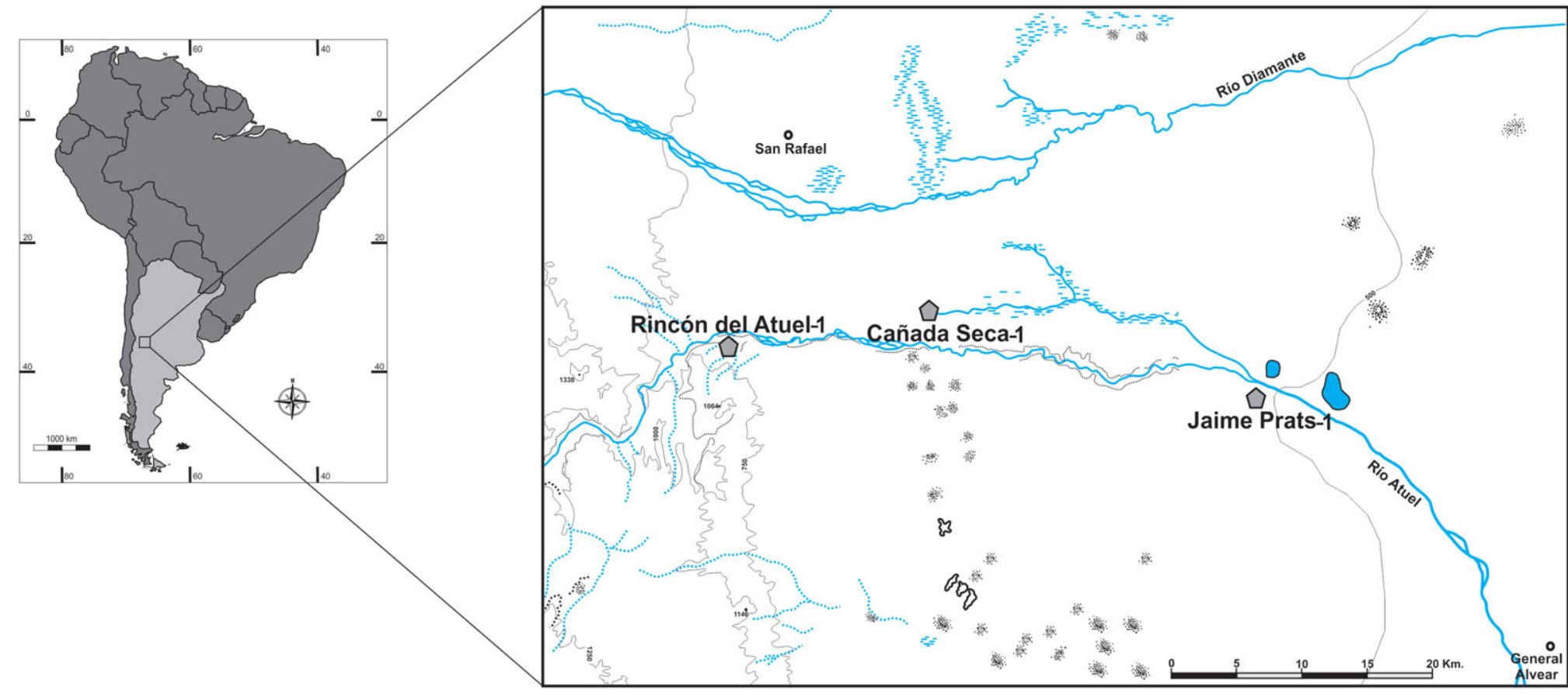

Figura 1. Valle medio del Río Atuel y ubicación de los sitios arqueológicos estudiados. (Color online) 
propuesta que, en zonas de transición entre caza-recolección y producción, la sola presencia de cultígenos no necesariamente implica agricultura, y que hay distintos mecanismos que explican el registro de plantas domésticas en una región, incluyendo tanto intercambio con vecinos productores como la producción local (Gil 1998). El registro arqueológico posterior a la denominada "fase productora inicial" fue atribuido a poblaciones agroalfareras (Lagiglia 2002), si bien se propuso que previo al contacto hispano-indígena las mismas habrían sido cazadoras-recolectoras. Esta interesante reversión temporal en los patrones de subsistencia no ha sido estudiada en detalle, como tampoco el nivel de agricultura propuesto para las ocupaciones posteriores a la fase inicial. Las evidencias arqueobotánicas sugieren que el Valle del Atuel habría sido el sector más meridional con relativamente alta densidad y diversidad de plantas domésticas prehispánicas (Gil et al. 2014a).

\section{Ambiente, Recursos y Ecología Isotópica en el Atuel Medio}

La cuenca media del Río Atuel se localiza entre aproximadamente $35^{\circ} 4^{\prime}$ S, $69^{\circ} 36^{\prime}$ O y $35^{\circ} 16^{\prime}$ S, 67³1' O (Figura 1). Fitogeográficamente, este tramo del río se desarrolla principalmente en el Monte, con un régimen de precipitación veraniego y un promedio de $250 \mathrm{~mm}$ anuales. Estudios arqueobotánicos destacan entre las especies silvestres el registro de Prosopis sp. $\mathrm{y}$, en menor abundancia, Geoffroea decorticans y otros taxones, aunque con una notoria subrepresentación (Giardina et al. 2015; Llano y Andreoni 2012). Entre las plantas domésticas, los estudios registran en forma más frecuente y abundante Zea mays, siguiendo Cucurbita sp., Phaseolus sp. y Chenopodium quinoa (Gil et al. 2014b). La información zooarqueológica señala una amplia diversidad taxonómica para el Holoceno tardío (Giardina et al. 2015; Gil y Neme 2010). Se destacan dasipódidos, huevos de reídos, peces y roedores, aunque estos últimos sin evidencias de consumo (Fernández 2012), y una baja representación de reídos y camélidos.

La ecología isotópica, basada sobre recursos de la región, ofrece valores para los recursos más significativos en el registro arqueológico y en el ecosistema actual, incluyendo peces, camélidos, maíz, dasipódidos, algarrobo y puma (ver debajo). Los trabajos de Gil y colaboradores (2013) y Ugan y colaboradores (2012) presentan el patrón de distribución espacial isotópica $\left(\delta^{18} \mathrm{O}\right)$ de las aguas para la región, marco de referencia necesario para entender los resultados de este isótopo en muestras óseas humanas. Estos datos se agrupan diferencialmente en cuatro áreas geográficas: alta cordillera de los Andes; piedemonte andino; ríos en tierras bajas (todos con aguas predominantemente de origen andino, pero con diferencia en la altitud); y un cuarto grupo constituido por Payunia-Nevado que incluye aguas de arroyos, vertientes y lagos de probable origen extra-andino (Texto Suplementario 1).

\section{Sitios y Contextos en el Atuel Medio}

El trabajo compara y articula los resultados generados a partir del estudio de los restos óseos humanos provenientes de los sitios Rincón del Atuel-1 (RA-1), Cañada Seca-1 (CS-1) y Jaime Prats-1 (JP-1; Figura 1 y Tabla Suplementaria 1). El sitio RA-1 se emplaza sobre una terraza aluvial (3445'31' S, 68 22' '14" O, a 747 m snm; Figura 1; Gil et al. 2008). Registra ocupaciones humanas entre los ca. 1700-1000 años aP (Gil et al. 2008). Este registro muestra dos unidades ocupacionales diferentes: una entre aproximadamente 1700-1500 años aP y otra entre 1500 1000 años aP. Se recuperaron cuatro individuos, todos ellos asignados a la ocupación más antigua. Dos individuos se encontraban enterrados en forma primaria, uno al lado del otro en forma casi paralela. Los otros dos se encontraban al lado de los anteriores, pero dispuestos como entierros secundarios (Gil et al. 2008). Junto con el material óseo humano se hallaron dos puntas de hueso como acompañamiento. Aunque se detectaron ocupaciones asociadas a contextos domésticos en las inmediaciones, estas no estaban vinculadas al evento de entierro. Un fechado radiocarbónico señala para este enterratorio una edad de $1760 \pm$ $70{ }^{14} \mathrm{C}$ aP (Tabla 1; Gil et al. 2008:Tabla 1).

El sitio CS-1 se emplaza en el valle medio del Río Atuel (3445'2.6”' S, 69¹'18.3'’ O, a 596 m snm; Figura 1). Recientes análisis han definido a CS-1 como un enterratorio múltiple mezclado, 
Tabla 1. Especímenes Óseos Humanos Adultos Analizados y sus Respectivos Isótopos $\left(\delta^{13} \mathrm{C}, \delta^{15} \mathrm{~N}, \delta^{18} \mathrm{O}\right)$.

\begin{tabular}{|c|c|c|c|c|c|c|c|c|c|}
\hline Sitio & Código & Código (fechado ${ }^{14} \mathrm{C}$ ) & Años ${ }^{14} \mathrm{C}$ aP & \pm & $\delta^{15} \mathrm{~N}$ & $\delta^{13} \mathrm{Ccol}$ & $\delta^{13} \mathrm{Cap}$ & $\delta^{18} \mathrm{Oap}$ & $\delta^{18} \mathrm{Oab}$ \\
\hline CS-1 & CS-10001 & LP-1374 & 1420 & 60 & 11,60 & $-15,70$ & -9 & $-5,4$ & $-8,16$ \\
\hline CS-1 & 10015 & - & - & - & 10,93 & $-14,56$ & $-9,51$ & $-5,96$ & $-8,88$ \\
\hline CS-1 & 66 & - & - & - & 10,41 & $-15,80$ & $-9,43$ & $-6,80$ & $-9,97$ \\
\hline CS-1 & 10007 & - & - & - & 12,65 & $-14,93$ & $-9,99$ & $-7,18$ & $-10,46$ \\
\hline CS-1 & 65 & - & - & - & 13,19 & $-15,32$ & $-10,39$ & $-7,33$ & $-10,66$ \\
\hline CS-1 & 61 & - & - & - & 11,49 & $-15,73$ & $-10,16$ & $-7,18$ & $-10,46$ \\
\hline CS-1 & 10013 & - & - & - & 10,99 & $-14,32$ & $-8,12$ & $-4,85$ & $-7,45$ \\
\hline CS-1 & 10004 & - & - & - & 11,68 & $-15,61$ & $-10,05$ & $-7,16$ & $-10,44$ \\
\hline CS-1 & 10012 & - & - & - & 10,91 & $-15,92$ & $-10,38$ & $-7,84$ & $-11,32$ \\
\hline CS-1 & REM-2 & - & - & - & 12,37 & $-15,27$ & $-10,78$ & $-7,10$ & $-10,36$ \\
\hline CS-1 & REM-3 & - & - & - & 12,59 & $-14,16$ & $-9,23$ & $-7,85$ & $-11,33$ \\
\hline CS-1 & REM-1 & - & - & - & 12,03 & $-14,70$ & $-9,46$ & $-6,78$ & $-9,95$ \\
\hline CS-1 & 10005 & - & - & - & 11,36 & $-14,39$ & $-9,53$ & $-7,65$ & $-11,07$ \\
\hline CS-1 & 10009 & - & - & - & 10,75 & $-14,87$ & $-9,42$ & $-7,91$ & $-11,41$ \\
\hline CS-1 & 10008 & - & - & - & 10,82 & $-14,83$ & $-9,62$ & $-6,04$ & $-8,99$ \\
\hline CS-1 & AF-2019 & - & - & - & 10,40 & $-14,50$ & $-10,1$ & $-3,7$ & $-5,96$ \\
\hline CS-1 & AF-2018 & - & - & - & 11,50 & $-14,30$ & $-9,8$ & $-2,8$ & $-4,79$ \\
\hline CS-1 & AF-2017 & - & - & - & - & $-14,90$ & - & - & - \\
\hline CS-1 & AF-2020 & LP-1184 & 1790 & 50 & 11,30 & $-14,30$ & $-9,5$ & $-2,5$ & $-4,40$ \\
\hline JP-1 & JP-1155 & - & - & - & 10,6 & $-16,8$ & $-10,2$ & $-6,7$ & $-9,84$ \\
\hline JP-1 & JP-1154 & - & - & - & - & - & - & - & - \\
\hline JP-1 & JP-1352 & AA-59589 & 1880 & 49 & 9,9 & $-16,3$ & $-10,6$ & $-5,8$ & $-8,67$ \\
\hline JP-1 & $\mathrm{JP} / \mathrm{J} 4$ & AA-59590 & 1887 & 42 & 9,8 & $-17,4$ & $-13,5$ & $-8,6$ & $-12,30$ \\
\hline JP-1 & JP-21 & - & - & - & - & $-17,9$ & - & - & - \\
\hline RA-1 & AF-500 & LP-1370 & 1760 & 70 & 10,3 & $-13,1$ & $-8,1$ & $-3,5$ & $-5,7$ \\
\hline RA-1 & AF-501 & - & - & - & 9,98 & $-15,04$ & $-9,69$ & $-3,66$ & $-5,96$ \\
\hline RA-1 & AF-502 & - & - & - & 8,85 & $-14,95$ & $-7,80$ & $-2,84$ & $-4,79$ \\
\hline RA-1 & AF-503 & - & - & - & 9,4 & $-13,8$ & $-7,9$ & $-3,7$ & $-5,96$ \\
\hline
\end{tabular}

donde la muestra se compone principalmente de elementos óseos humanos aislados semejante a un osario de tipo secundario (Peralta 2015). Se obtuvo un NMI de 23 individuos, que incluye adultos, juveniles y subadultos. En este sitio no se hallaron contextos domésticos, quedando su funcionalidad restringida a la funebria. Acompañando los restos humanos se hallaron adornos (cuentas de turquesa y tembetás) y ocho puntas de proyectil. Las fechas radiocarbónicas de CS1 dieron una cronología que se extiende desde 1800 a 1400 años ${ }^{14} \mathrm{C}$ aP (Tabla 1).

El sitio JP-1, excavado en 1988 (Lagiglia 1994), se emplaza próximo a la barranca del Río Atuel, en las proximidades de la localidad homónima (Figura 1). Se calculó un NMI de 125 individuos, de los cuales cuatro se hallaron en posición primaria (Lagiglia 1994). El resto de los individuos se encontraban mezclados y disociados, similar al contexto observado en CS1. Este sitio también tiene una funcionalidad exclusiva de funebria, no asociada con ocupa- ciones domésticas. Se encontraron tres puntas de proyectil como material cultural asociado a los restos óseos humanos. Los fechados ubican estos restos entre ca. 2050 y 1750 años ${ }^{14} \mathrm{C}$ aP (Tabla 1; Gil y Neme 2010).

La Figura 2 señala comparativamente la estructura cronológica en años calibrados de los registros de JP-1, RA-1 y CS-1 (Tabla 1; Gil y Neme 2010). El más antiguo, JP-1, tiene una fecha promedio de $1836 \pm 24 \mathrm{aP}$; los rangos $1 \delta$ de los 5 fechados procedentes del sitio están comprendidos entre 2145 y 1536 años cal aP. Este registro es seguido temporalmente por RA-1, cuya única fecha es $1760 \pm 70 \mathrm{aP}$, con rango $1 \delta$ entre 1707 y 1545 años cal aP. Finalmente, CS-1 tiene fecha media de $1594 \pm 24 \mathrm{aP}$; los rangos $1 \delta$ de los 4 fechados procedentes del sitio están comprendidos entre 1701 y 1189 años cal aP. Con base en la suma de probabilidades para cada sitio, los rangos $1 \delta$ de conjuntos se encuentran entre 1869 años cal aP y 1189 años cal aP, indicando un rango de ocupaciones de 680 


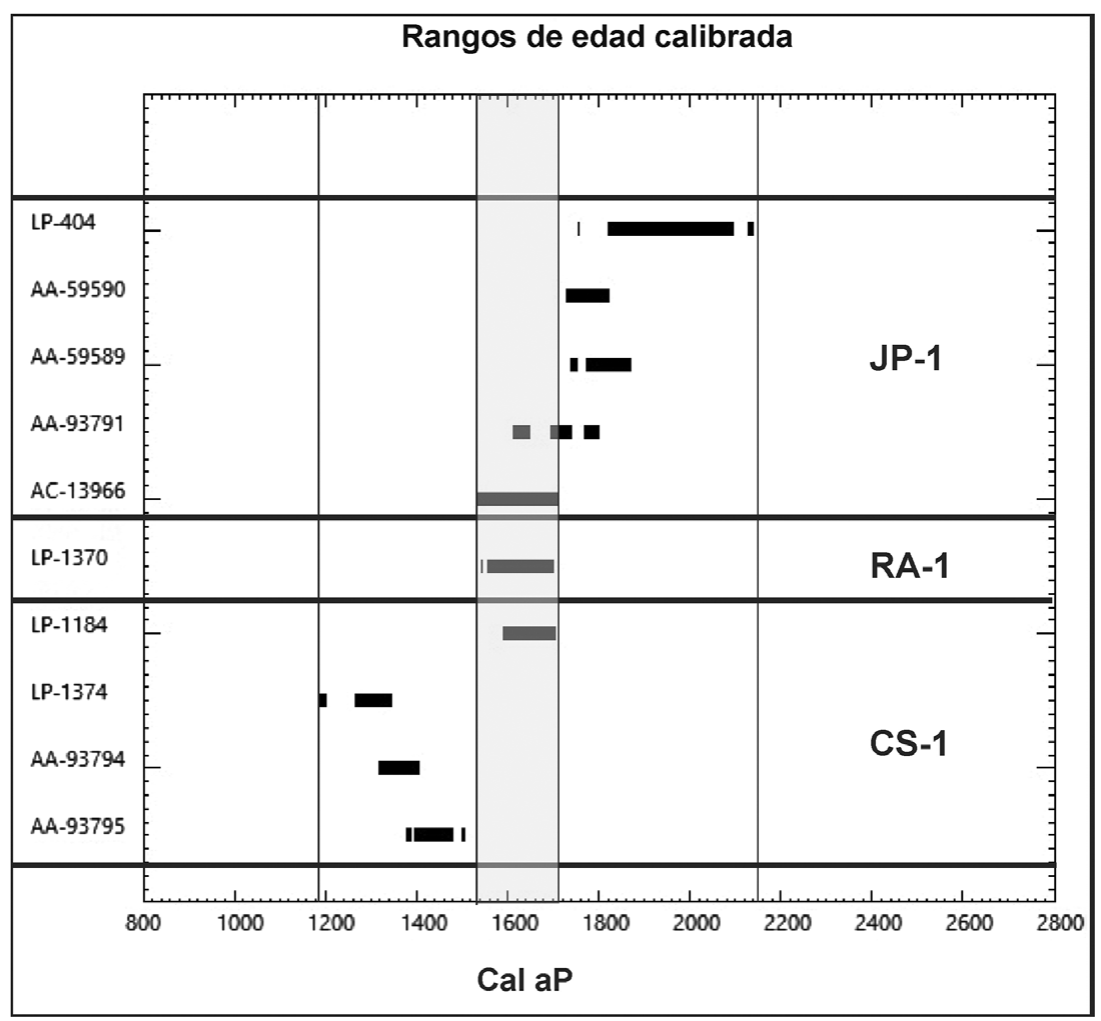

Figura 2. Fechados radiocarbónicos calibrados con CALIB 7.1 (Stuiver et al. 2017) sobre restos humanos de los sitios JP-1, RA-1 y CS-1. EI sector en gris indica el sobrelapamiento temporal máximo de los tres sitios.

años. Considerando las fechas individualmente, el rango va desde 2145 a 1189 años cal aP, lo que implica un lapso ocupacional de 956 años. También usando un desvío estándar se observa que los tres conjuntos podrían tener entierros contemporáneos, principalmente entre 1710 años cal aP y 1593 años cal aP, durante aproximadamente 117 años.

\section{Aspectos Metodológicos}

\section{Isótopos Estables $\left({ }^{13} \mathrm{C},{ }^{15} \mathrm{Ny}{ }^{18} \mathrm{O}\right)$}

Se presentan resultados de los análisis de isótopos estables de ${ }^{13} \mathrm{C}$ y ${ }^{15} \mathrm{~N}$ basados en el procesamiento de la fracción orgánica (colágeno) y de ${ }^{13} \mathrm{Cy}{ }^{18} \mathrm{O}$ obtenidos de la fracción inorgánica (hidroxiapatita o apatita). Los valores $\delta^{13} \mathrm{C}$ y $\delta^{18} \mathrm{O}$ se calcularon utilizando el estándar V-PDB, mientras que el $\delta^{15} \mathrm{~N}$ se calculó con el estándar AIR. El colágeno y la hidroxiapatita de material óseo se obtuvieron procesando las muestras en el Laboratorio de Paleoecología Humana del IANIGLA-Grupo Vinculado San Rafael, siguiendo el protocolo descripto en Coltrain y Leavitt (2002). Las mediciones de los isótopos estables de $\delta^{13} \mathrm{C}, \delta^{15} \mathrm{~N}$ y $\delta^{18} \mathrm{O}$ se efectuaron en SIRFER de la University of Utah (código MSR) y en University of South Florida (código USF). Para evaluar la integridad de las muestras se analizó en cada una de ellas la relación C:N siguiendo los criterios de Ambrose (1990), aceptándose aquellas cuyos valores estén comprendidos entre 2,9 y 3,6. Para el fraccionamiento trófico se utiliza para el $\delta^{15} \mathrm{~N}$ un enriquecimiento de $4 \%$, y para $\delta^{13} \mathrm{C}$ se estima un enriquecimiento del colágeno óseo respecto al recurso de $1 \%$ (Ambrose 1990).

Los isótopos de oxígeno $\left({ }^{18} \mathrm{O} /{ }^{16} \mathrm{O}\right)$ se utilizan principalmente para discutir movilidad y patrones de residencia (Dupras y Schwarcz 2001; Sponheimer y Lee-Thorp 1999). Estos isótopos se registran en el fosfato y carbonato de la hidroxiapatita, y reflejan principalmente la 
composición isotópica del agua corporal, determinada básicamente por el agua ingerida, en el momento de formación de los huesos y dientes (Sponheimer y Lee-Thorp 1999). A su vez, el agua bebida varía de modo regular en relación a la latitud, elevación, patrones de lluvia y otros factores ambientales (Dansgaard 1964; Gat 1996). Para la región se cuenta con estudios previos que permiten comparar los valores obtenidos de hueso humano con un marco espacial de variación de $\delta^{18} \mathrm{O}$ en las aguas (Gil et al. 2014a; Ugan et al. 2012).

Para entender las tendencias en las dietas humanas inferidas desde los isótopos se requiere un modelo sobre las cadenas tróficas que contemple la variabilidad isotópica de los recursos de base (Szpak et al. 2013). Por ello se utilizaron los datos ecológicos disponibles en la región (Giardina et al. 2014; Gil et al. 2014a; Tabla Suplementaria 2).

Para la interpretación de la dieta se utilizaron modelos bayesianos de mezcla de dieta. Estos modelos permiten ajustar la interpretación de las dietas profundizando el análisis bivariado mediante modelos de combinación estadística donde se explora la probabilidad de los aportes de distintos recursos (Bernal et al. 2016; Giardina et al. 2014; Moore y Semmens 2008; Parnell et al. 2013; Phillips 2012; Phillips et al. 2014). Para este análisis se utilizó MixSIAR (Stock y Semmens 2013; Texto Suplementario 2).

\section{Morfometría Craneofacial}

Se definieron 102 puntos anatómicos, 62 en el cráneo y 40 en la mandíbula. Los 62 puntos del cráneo fueron registrados como coordenadas en tres dimensiones utilizando un brazo mecánico Microscribe G2X, mientras que los 40 puntos de las mandíbulas fueron digitalizados en dos dimensiones con el programa tpsDIG 1.20, a partir de imágenes fotográficas obtenidas mediante una cámara digital Canon Power Shot SX10 IS (Texto Suplementario 2).

\section{Morfometría Dental}

El análisis de la variación odontométrica de JP-1, RA-1 y CS-1 se basó en los diámetros cervicales mesio-distal (MD) y vestíbulo-lingual (VL) de 37 individuos con dentición adulta. La toma de datos se realizó con un calibre digital de puntas finas Paleo Tech Hillson-Fitzgerald Dental Caliper (Hillson et al. 2005), siguiendo los criterios propuestos por Hillson y colaboradores (2005) y las modificaciones propuestas por Bernal (2008). Las mediciones fueron registradas empleando el método side count (Scott 2008), en primeros premolares $\left(\mathrm{PM}^{1}\right)$ y primeros molares $\left(\mathrm{M}^{1}\right)$ de la arcada dental superior, priorizando las piezas izquierdas cuando se encontraban presentes. Los datos faltantes fueron estimados utilizando el método de imputación múltiple propuesto por Schafer (1999a, 1999b). Posteriormente, los datos fueron transformados para obtener variables de forma y de tamaño dental, las cuales fueron empleadas para establecer distancias biológicas (variables de forma) mediante análisis de componentes principales y análisis de conglomerados, y evaluar la existencia de relaciones significativas entre los sistemas de subsistencia de los grupos analizados y el tamaño dental de sus individuos (mediante ANOVA). En el Texto Suplementario 2 se detalla el conjunto de series craneanas estudiadas y la metodología estadística aplicada en el análisis de datos.

\section{Asociación entre Variación Craneofacial-Dental y Diversidad Isotópica}

Se realizaron regresiones espaciales entre las variables morfométricas craneofaciales y dentales (tamaño y forma del cráneo completo, forma del esqueleto facial, forma de la bóveda, forma de la base craneana, tamaño dental, forma dental). También se efectuaron estos análisis para variables isotópicas $\left(\delta^{15} \mathrm{~N}, \delta^{13} \mathrm{C}_{\mathrm{ap}}, \delta^{13} \mathrm{C}_{\mathrm{col}}\right)$ a partir de los valores medios de cada muestra (Texto Suplementario 2).

\section{Resultados}

\section{Tendencia Isotópica $\left({ }^{13} \mathrm{C},{ }^{15} \mathrm{~N}\right)$}

Los tres sitios arqueológicos cuentan con datos de isótopos estables $\left(\delta^{13} \mathrm{C}, \delta^{15} \mathrm{~N}\right)$ obtenidos sobre colágeno y apatita de huesos humanos (Gil y Neme 2010; Gil et al. 2010; datos no publicados previamente; Tabla 1). La Figura 3 se basa en los datos procedentes de individuos adultos y muestra las tendencias por sitio e individuos. Los valores medios de $\delta^{13} \mathrm{C}$ para CS-1 y RA-1 son 


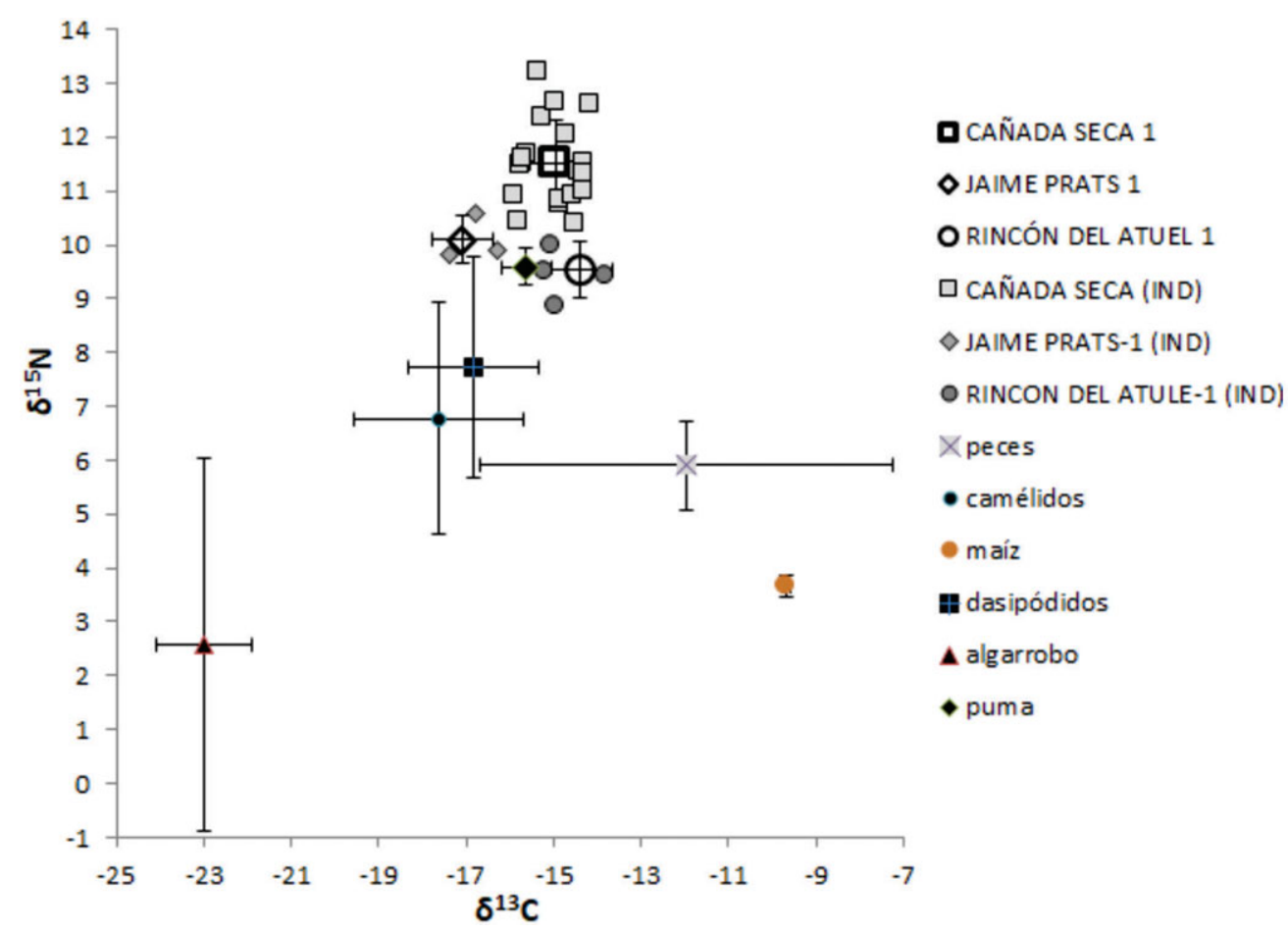

Figura 3. Tendencia por individuo y promedios por sitio en los valores de $\delta^{13} \mathrm{C}$ y $\delta^{15} \mathrm{~N}$ comparados con las tendencias para los recursos, adecuando estos valores por fraccionamiento isotópico. (Color online)

similares $(-14,9 \%$ y $-14,4 \%$ o respectivamente) y significativamente más enriquecidos que la media de JP-1 (-17,1\%o). La misma tendencia se nota en el $\delta^{13} \mathrm{C}_{\text {ap }}$ donde los valores medios de CS-1 y RA-1 son similares (-8,3\% y $-9 \%$ respectivamente) mientras que JP-1 presenta valores menores $(-11,4 \%)$. Por otra parte, los valores medios de $\delta^{15} \mathrm{~N}$ para RA-1 y JP-1 son similares ( $9,5 \%$ y $10,1 \%$ respectivamente) y menores que el de CS-1 (11,5\%o). Considerando la ecología isotópica (Tabla Suplementaria 2) se observan diferencias notorias entre los tres sitios. Con base en estas tendencias se propone un incremento en la proporción de recursos de alto nivel trófico en las dietas humanas de CS-1 respecto a JP-1 y RA-1, siendo éstas últimas las de valores menores. Asimismo, se nota un incremento en la proporción de recursos $\mathrm{C}_{4}$ (consumidos directa o indirectamente) en RA-1 y CS-1 respecto a JP-1. A nivel individual no se registra solapamiento entre los valores isotópicos de los sitios, lo que fortalece la diferencia entre los conjuntos. Aplicando el test de Mann-Whitney, con un límite de $p=0,05$, se determinó que estas diferencias fueron significativas en todas las comparaciones, excepto en el caso de los valores de $\delta^{15} \mathrm{~N}$ entre RA-1 y JP-1.

La Figura 3 muestra la relación en las tendencias isotópicas de los recursos potenciales más significativos que podrían haber conformado la dieta de los individuos de los tres sitios, usando como referencia el registro arqueológico del valle medio del Atuel (Giardina et al. 2015; Gil y Neme 2010). De este modo se comparan los valores isotópicos humanos $\left(\delta^{13} \mathrm{C}\right.$ y $\delta^{15} \mathrm{~N}$; promedio por sitio e individuos segregados por sitio) con los promedios y desvío estándar de pumas, peces, camélidos, dasipódidos, algarrobo y maíz (Giardina et al. 2014; Gil y Neme 2010; Gil et al. 2011; Tabla Suplementaria 2). Mientras los recursos presentan valores medios de $\delta^{15} \mathrm{~N}$ entre 2,6\% y 9,6\% (Tabla Suplementaria 2) los humanos de CS-1 y JP-1 presentan valores superiores a los recursos, incluyendo los carnívoros, en tanto que RA-1 presenta valores menores que los humanos de esos sitios (promedio 9,6\% con extremos entre $8,8 \%$ y $9,9 \%$; Tabla 1 ), y semejantes a los de los carnívoros. Sobre el valor medio de 
estos últimos (los recursos con $\delta^{15} \mathrm{~N}$ más alto), los humanos de CS-1 se incrementan hasta 3,6\%o y los de JP-1 hasta 1\%, es decir que en CS1 se registra un nivel superior en la cadena trófica respecto de los carnívoros, de acuerdo con el promedio del "efecto del nivel trófico" generalmente aceptado (Figura 3). Respecto del promedio, el incremento es de 1,9\% para CS-1, y de $0,5 \%$ en JP-1 (Figura 3 ).

Los valores medios de $\delta^{13} \mathrm{C}$ de los recursos varían entre $-23 \%$ y $-9,6 \%$. Este último valor corresponde al maíz, propuesto como el recurso doméstico más significativo en la dieta humana (Gil et al. 2014b). Los valores $\delta^{13} \mathrm{C}$ para humanos adultos de CS-1 varían entre $-14,1 \%$ y $-15,9 \%$, con un promedio de $-14,9 \%$. Por lo tanto, considerando los mecanismos fotosintéticos generales, estos se emplazarían en una dieta mixta de recursos $\mathrm{C}_{3}$ (ejemplificados en la figura por algarrobos) y $\mathrm{C}_{4}$ (como el maíz). Comparando con la fauna considerada, los valores son semejantes a los del carnívoro, quedando comprendidos entre los de camélidos, dasipódidos y peces (Figura 3). Por ello, también es necesario considerar el consumo de fauna que tenga enriquecido su $\delta^{13} \mathrm{C}$, y no explicar el enriquecimiento de éste isótopo solamente por consumo de plantas $\mathrm{C}_{4}$ como el maíz. Una tendencia similar en este isótopo se encuentra en los individuos de RA-1 (promedio de 14,4\%o), aunque el $\delta^{15} \mathrm{~N}$ más bajo podría señalar que el enriquecimiento en ellos se debe a un mayor aporte vegetal $\mathrm{C}_{4}$ que en CS-1. Para JP-1, el valor promedio de $-17 \%$ refleja que el aporte vegetal $\mathrm{C}_{4}$ fue muy bajo o nulo. Esta información sobre los isótopos estables de los recursos señala que, si bien el maíz pudo formar parte de la dieta, no fue un recurso cuantitativamente significativo en CS-1 y menos aún en JP-1. Esto se basa primero en las diferencias tróficas entre los vegetales y humanos en estos dos sitios, según lo señalan los valores de $\delta^{15} \mathrm{~N}$ (8\% de diferencia respecto a los valores medios de CS-1, 6,6\% respecto a JP-1 y $6 \%$ con RA-1) y segundo en los valores significativamente menores en $\delta^{13} \mathrm{C}$ de estos respecto a los del maíz (diferencia de 5,2\%o con CS-1/RA-1 y 7,4\% en JP-1). Esta discusión es retomada al mostrar los resultados de la aplicación de modelos de mezcla bayesianos para modelar la dieta humana.

\section{Tendencia Isotópica $\left({ }^{18} \mathrm{O}\right)$}

Los valores esperados para aguas bebidas de $\delta^{18} \mathrm{O}$ en material óseo humano varían en JP-1 entre $-8,6 \%$ y $-12,3 \%$, en RA-1 entre $-4,8 \%$ o y $-5,7 \%$, mientras que en CS-1 el rango va desde $-4,4 \%$ a $-11,4 \%$ (Figura Suplementaria 1). La Figura 4 señala la variación de éste isótopo en cada sitio y muestra que la tendencia en RA-1 se separa significativamente respecto de las tendencias en JP-1 y CS-1, mientras estas se asemejan. Así, RA-1 muestra valores más altos y menor variabilidad entre individuos que JP-1 y CS-1, que muestran mayor variabilidad entre individuos y valores más bajos. La Figura Suplementaria 1 muestra los rangos de valores de las aguas superficiales según cada región geográfica (Gil et al. 2014a; Ugan et al. 2012) y los valores estimados de aguas bebidas por cada individuo según su valor de $\delta^{18} \mathrm{O}$. Los individuos de RA-1 señalan una semejanza con aguas de Payunia-Nevado, mientras que JP-1 y CS-1 muestran semejanza con esta región, pero también con el piedemonte. Esto indica para estos dos últimos sitios una mayor diversidad de rangos o rangos más amplios que en RA-1. Un número de muestras, principalmente de CS-1, presentan valores intermedios entre ambos sectores, lo que podría señalar un promedio en el uso de los mismos ya que no se cuenta con aguas que específicamente tengan ese patrón de $\delta^{18} \mathrm{O}$ $(-12 \%$ a $-9 \%$ ).

\section{Modelo de Mezcla de Dieta}

Teniendo en cuenta el registro zooarqueológico y arqueobotánico de la región (Gil y Neme 2010), se utilizaron los recursos más ubicuos y por ende los aceptados como más significativos a nivel regional. Sobre esta base, se comparan los aportes a la dieta humana del Atuel medio de camélidos, dasipódidos, peces, algarrobo, y maíz (Tabla Suplementaria 2). Los resultados del modelo señalan para la fracción de colágeno un aporte significativamente mayor de maíz en RA1 (aunque tiene significativa correlación negativa con peces) respecto a JP-1 y CS-1. Por su parte, los camélidos y peces no muestran grandes variaciones entre los tres sitios, aunque estas diferencias sí son notorias en el algarrobo que habría sido menos importante para CS-1 que 


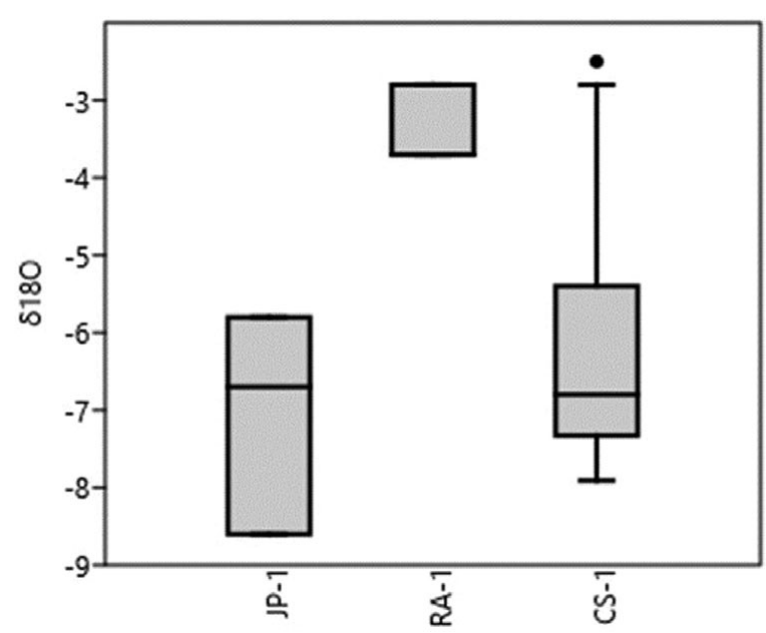

Figura 4. Tendencia estadística por sitio en valores de $\delta^{18} \mathrm{O}$ obtenidos sobre muestras óseas humanas.

Tabla 2. Frecuencia de Recursos en la Dieta de los Tres Sitios

\begin{tabular}{llcc}
\hline Sitios & Recursos & $\begin{array}{c}\text { Proteínas } \\
\text { (colágeno) }\end{array}$ & $\begin{array}{c}\text { Dieta Total } \\
\text { (apatita) }\end{array}$ \\
\hline CS-1 & Camélido & $19 \%$ & $28,2 \%$ \\
& Dasipódidos & $56 \%$ & $52,20 \%$ \\
& Peces & $10,3 \%$ & $3,8 \%$ \\
& Algarrobo & $3 \%$ & $11 \%$ \\
& Maíz & $8,1 \%$ & $2,2 \%$ \\
\hline JP-1 & Camélido & $25,5 \%$ & $14,4 \%$ \\
& Dasipódidos & $26,7 \%$ & $13,2 \%$ \\
& Peces & $9,9 \%$ & $42,8 \%$ \\
& Algarrobo & $23,1 \%$ & $12 \%$ \\
& Maíz & $7,8 \%$ & $5,1 \%$ \\
\hline RA-1 & Camélido & $21,4 \%$ & $12,4 \%$ \\
& Dasipódidos & $21,1 \%$ & $9,4 \%$ \\
& Peces & $12,9 \%$ & $7,5 \%$ \\
& Algarrobo & $16,1 \%$ & $52,2 \%$ \\
& Maíz & $23,6 \%$ & $3,1 \%$ \\
\hline
\end{tabular}

Nota: Resumen estadístico que señala frecuencia, en mediana, de recursos en la dieta grupal de cada sitio. Los pares de grises señalan recursos con correlaciones negativas superiores a $-0,60$ que no permiten segregar

significativamente los aportes entre ellos.

en los otros dos sitios (Tabla 2; Figura Suplementaria 2). Asimismo, la dieta total basada en apatita ósea presenta un patrón diferente, donde el maíz muestra valores bajos y similares entre los tres sitios, sin superar el 10\%. En CS-1 se observa mayor importancia de camélidos y dasipódidos, tanto a nivel proteico como de dieta total, que en los otros dos sitios. En RA-1 se destaca una significativamente mayor frecuencia de algarrobo que en CS-1 y JP-1 (Tabla 2; Figura Suplementaria 2).

\section{Variación Morfométrica Craneofacial}

Debido a las condiciones de preservación, del total de elementos anatómicos disponibles fue posible analizar 6 cráneos y 6 mandíbulas de individuos adultos (5 masculinos y 1 femenino; Tabla Suplementaria 1) para caracterizar la variación morfológica del Atuel medio. Dado que el tamaño muestral es bajo, se presentan análisis estadísticos descriptivos para evaluar la variación de la muestra disponible, que probablemente no represente de forma significativa la variación poblacional, pero permite caracterizar la variación de los individuos disponibles y las tendencias generales entre los tres sitios arqueológicos. En la Figura 5 se puede observar la variación en tamaño del cráneo de los individuos procedentes de los tres sitios arqueológicos estudiados. En relación al tamaño del cráneo (Figura 5a), los individuos de CS-1 presentan los cráneos más grandes, mientras que los individuos de RA-1 presentan los cráneos más pequeños, y los individuos de JP-1 cráneos de tamaño intermedio. La variación en tamaño de los individuos de CS-1 y RA-1 se solapa mínimamente entre sí, representando los dos extremos de variación de la muestra. Los tamaños craneales de los individuos de JP-1 se asemejan más a los de CS1 que a los de RA-1. Respecto al tamaño de la 

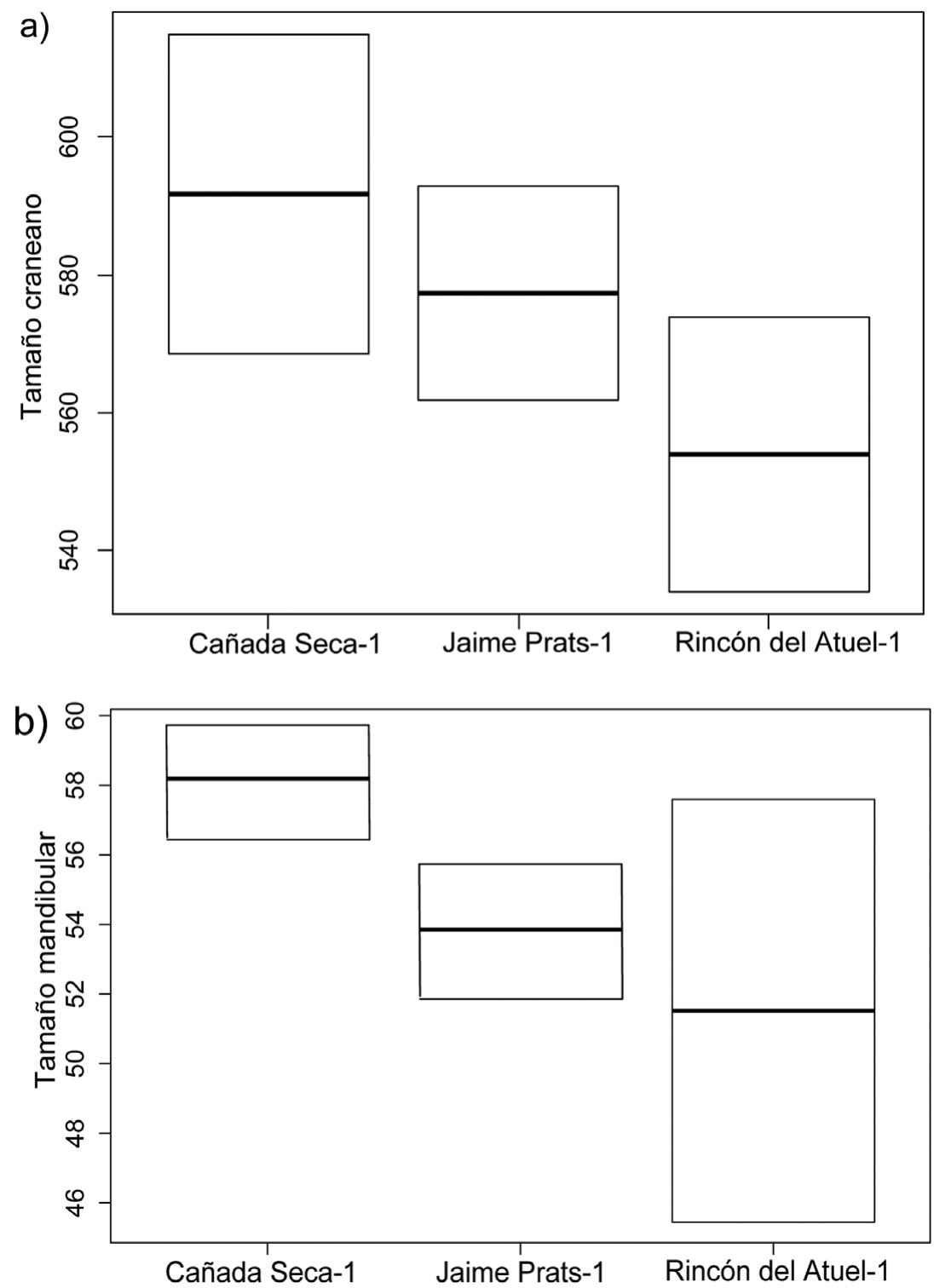

Figura 5. Diagramas de caja: (a) tamaño del cráneo y (b) tamaño mandibular.

mandíbula (Figura 5 b) se observa una tendencia de variación similar a la del cráneo, donde los individuos de CS-1 presentan las mandíbulas de mayores dimensiones, seguidos de JP-1 y finalmente las mandíbulas más pequeñas son las de RA-1. Sin embargo, a diferencia del cráneo, los tamaños de las mandíbulas de JP-1 se solapan con los de RA-1, en lugar de CS-1.

La Figura 6 muestra la variación en forma de los individuos analizados respecto al cráneo completo, esqueleto facial, bóveda, base craneana y mandíbula. A partir del análisis de componentes principales (ACP) del cráneo completo (Figura 6a) se puede ver que los individuos se agrupan por sitio arqueológico a lo largo del primer componente principal (CP). Los dos primeros $\mathrm{CP}$ resumen más del $60 \%$ de la variación en forma. Mientras que los individuos de JP-1 se ubican en el extremo negativo del CP1, los individuos de RA-1 se encuentran hacia el extremo positivo del CP1, y los individuos de CS-1 en una posición intermedia. Se observa 
a) Cráneo completo

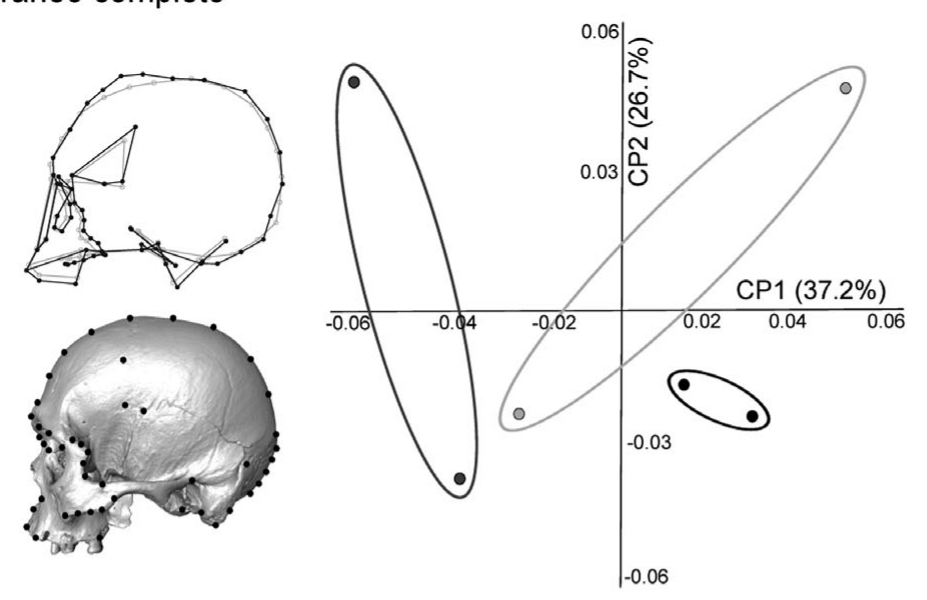

b) Esqueleto facial
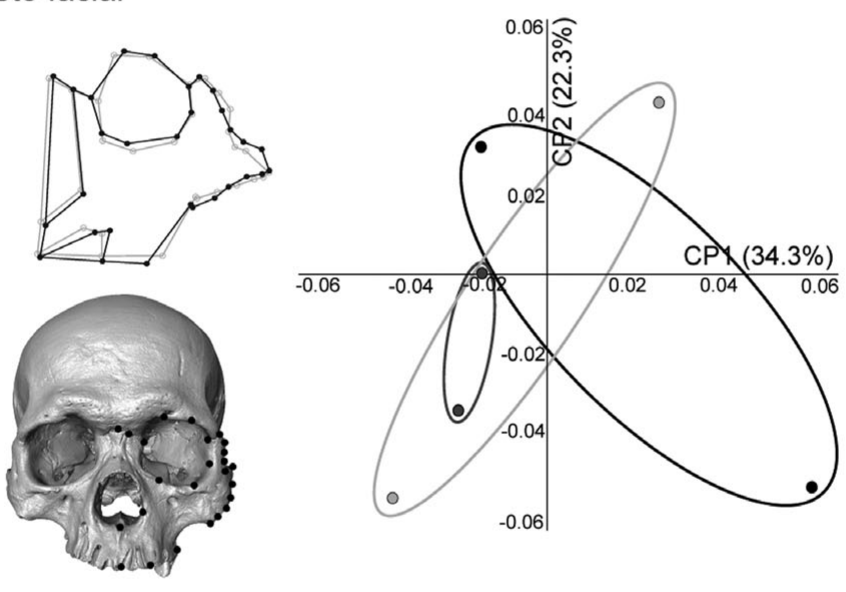

c) Bóveda
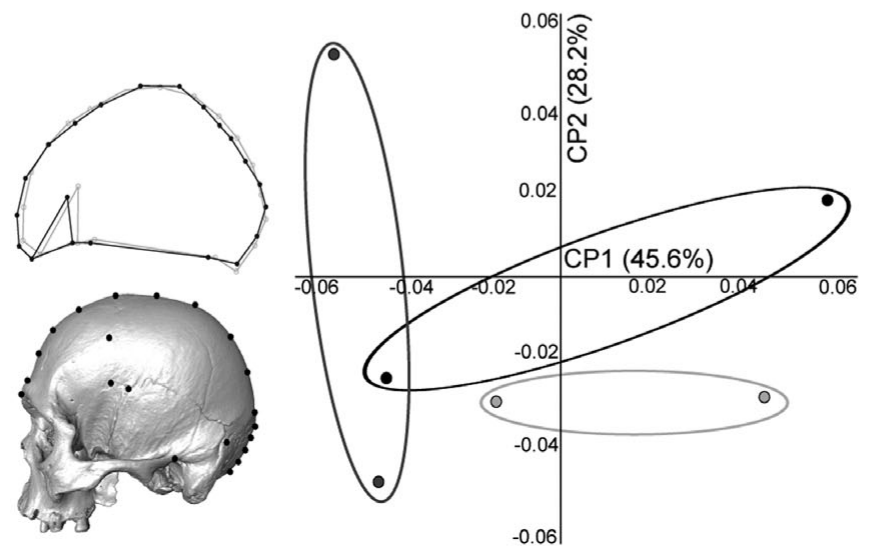

- Cañada Seca-1

- Jaime Prats-1

- Rincón del Atuel-1
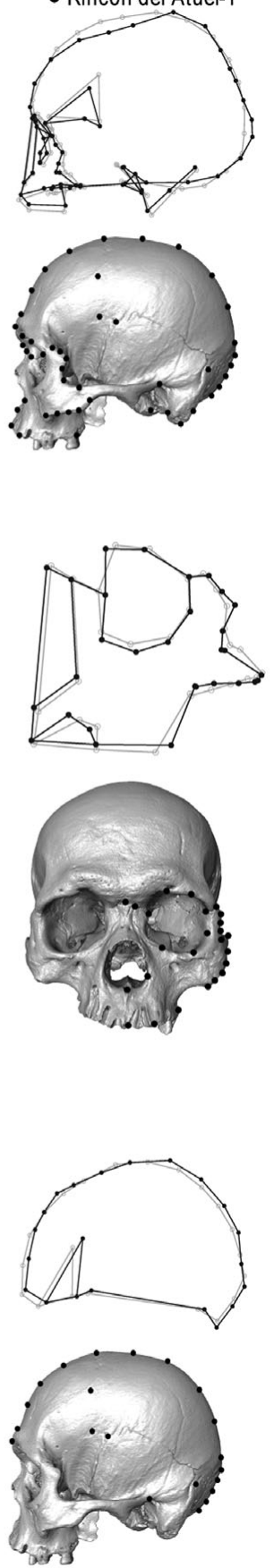

Figura 6. Análisis de Componentes Principales basados en la morfometría craneal y mandibular. 
d) Base craneana
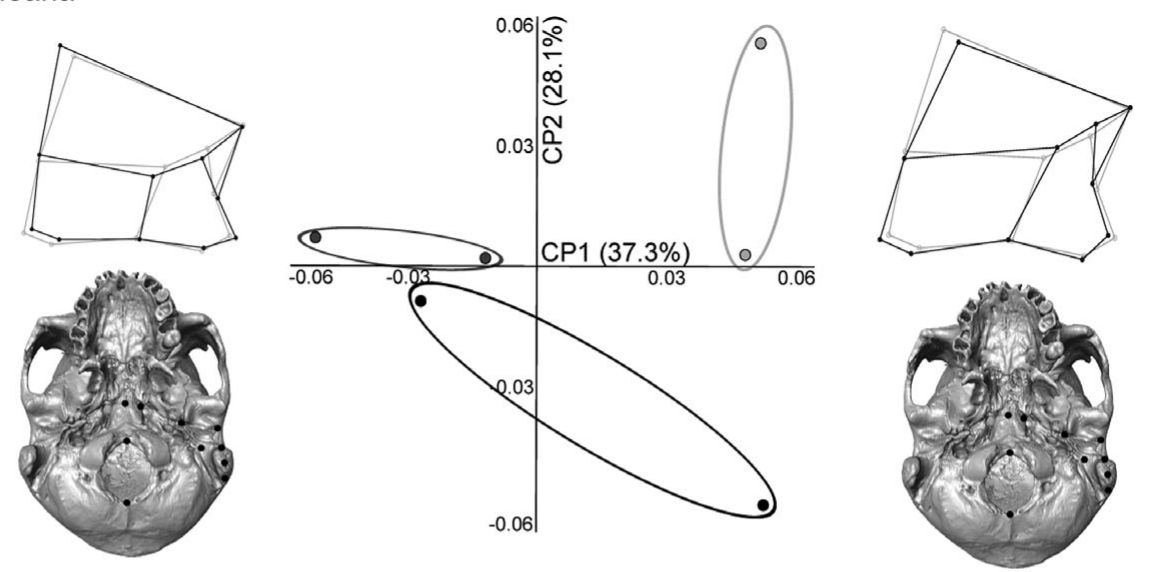

e) Mandíbula
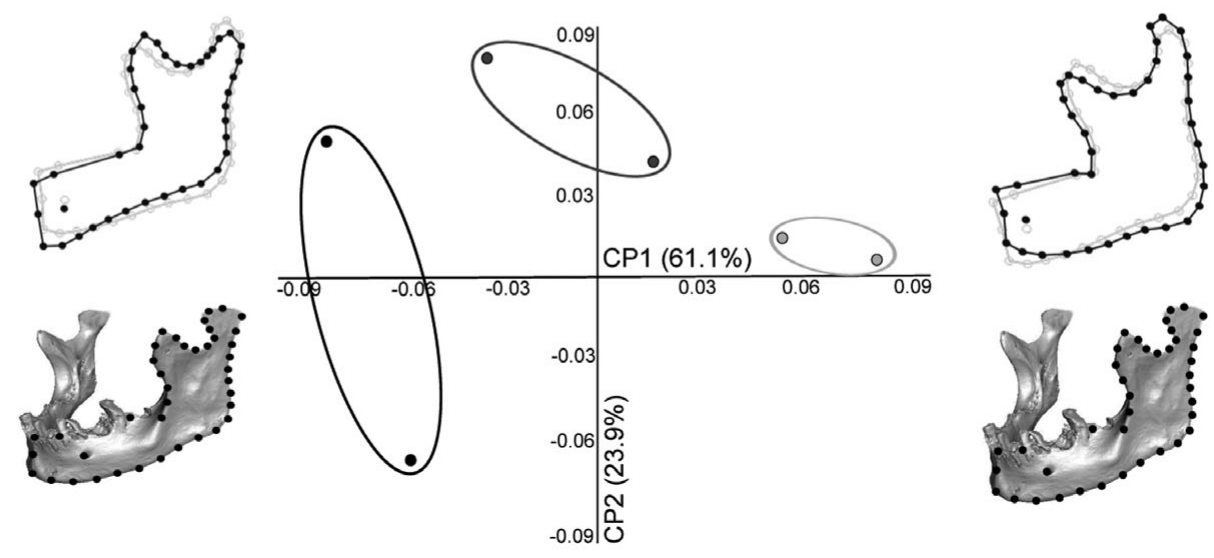

Figura 6. Continuación.

una importante amplitud de variación en los individuos de CS-1, uno de los cuales se acerca a los de JP-1, mientras que el otro se acerca a los individuos de RA-1. A partir de los wireframes se puede observar los rasgos que mayormente contribuyen a diferenciar a los individuos entre sí y a generar el patrón de variación que se observa en los extremos. Mientras que los individuos de JP-1 se caracterizan por presentar cráneos redondeados, con bóvedas más altas, procesos mastoideos con mayor proyección posterior y maxilares con una mayor proyección anterior, los individuos de RA-1 presentan bóvedas más alargadas en sentido antero-posterior, procesos mastoideos y maxilares menos desarrollados, y un aplanamiento en la región lámbdica como producto de prácticas culturales que modifi- caron el cráneo. Cabe destacar que uno de los individuos de CS-1 (AF-2019) comparte estas características junto con los individuos de RA-1. Es decir, algunos individuos presentan modificaciones culturales del cráneo, especialmente en la región lámbdica, mientras que otros no lo presentan, lo cual, con base en trabajos previos (Menéndez y Lotto 2016), coincide con las expectativas para la región.

La variación del esqueleto facial señala que las muestras se encuentran en gran parte superpuestas (Figura 6b), si bien uno de los individuos de CS-1 se encuentra en el extremo negativo, y uno de los individuos de RA-1 en el extremo positivo del primer componente principal. Las muestras de JP-1 se encuentran en el extremo negativo del primer componente 
principal, junto con las de CS-1. La variación de la bóveda craneana (Figura 6c) muestra que los individuos se distribuyen de acuerdo a la presencia o ausencia de modificaciones culturales del cráneo. Los individuos de JP1 se encuentran en el extremo negativo del primer componente principal; los mismos se caracterizan por no presentar modificaciones culturales del cráneo. Por otro lado, los individuos de RA-1 y CS-1 presentan modificaciones culturales del cráneo y se encuentran hacia el extremo positivo del primer componente principal. La base craneana (Figura 6d), al igual que el ACP del cráneo completo, muestra que los individuos se agrupan de acuerdo al sitio arqueológico de proveniencia, de manera que hacia el extremo negativo del primer componente principal se encuentran los individuos de JP-1, los individuos de RA-1 se localizan en una posición intermedia y hacia el extremo negativo del primer componente principal se ubican los individuos de CS-1. La Figura 6e muestra la variación en forma de la mandíbula mediante un análisis de componentes principales junto a los wireframes que muestran la variación a lo largo de los extremos positivo y negativo del primer componente principal. Los dos primeros componentes principales explican el $84 \%$ de la variación en forma mandibular. Se puede observar que las muestras se distribuyen geográficamente, es decir agrupándose de acuerdo con los sitios arqueológicos de procedencia. Los individuos de RA-1 se ubican en el extremo negativo del CP1, presentando un cuerpo mandibular más delgado $\mathrm{y}$ alargado antero-posteriormente, y una rama mandibular inclinada posteriormente, que forma un ángulo obtuso con el cuerpo mandibular. Las muestras de JP-1 se encuentran en una posición intermedia y las muestras de CS-1 en el extremo positivo del CP1, caracterizadas por presentar un cuerpo mandibular más grueso y una rama mandibular más alargada en sentido dorsoventral, que forma un ángulo recto con el cuerpo mandibular.

\section{Variación Odontométrica}

El ACP realizado con las variables de forma de los individuos de los sitios JP-1, RA-1 y CS-1 permitió establecer que los dos primeros componentes principales (CP) dan cuenta del $79 \%$ de la variación observada (Figura 7a). Los coeficientes de ponderación obtenidos para las variables de forma (Tabla Suplementaria 3), permiten establecer que el diámetro mesio-distal del $\mathrm{M}^{1}$ es la variable que más contribuye a diferenciar las muestras en el CP1, y que los diámetros vestíbulo-linguales del $\mathrm{PM}^{1}$ y el $\mathrm{M}^{1}$ hacen lo propio para el CP2; esto se ve claramente en el diagrama de dispersión biespacial (Figura 7a). Dicha figura también permite observar una mayor dispersión de los individuos de JP-1 en el CP1, mientras que los individuos de CS1 y RA-1 se disponen en torno al origen de coordenadas para dicho componente, con la excepción de uno de CS-1 que se ubica en el extremo positivo del CP1 y uno de RA-1 que presenta valores negativos intermedios. En el CP2 los individuos de JP-1 varían entre los valores positivos y negativos medios, mientras que los individuos de CS-1 presentan valores positivos y negativos más próximos al origen de coordenadas. Los individuos de RA-1 son los que presentan mayor dispersión sobre el CP2, ubicándose entre los valores positivos medios y el extremo negativo del CP2.

El análisis de conglomerados elaborado a partir de los valores obtenidos para CP1 y CP2 (Figura 7b) permitió agrupar a los individuos de CS-1 y JP-1 en una misma concentración, diferenciándolos de los individuos de RA-1. Por su parte, el coeficiente de correlación cofenética presentó un valor de 0,966 , reflejando una alta correlación entre las distancias evidenciadas en el dendrograma y las distancias originales calculadas entre individuos (ACP).

El análisis del factor tamaño dental mediante ANOVA de una vía dio un $F=14,76$ y un $p<0,0001$ (Tabla Suplementaria 4). La prueba post hoc de Di Rienzo, Guzmán, Casanoves (DGC) permitió definir que CS-1 se diferencia del resto de las muestras (Tabla Suplementaria 4), presentando los mayores tamaños dentales. Por su parte, el resto de las series, si bien se agrupan entre sí, se ordenan de forma decreciente en función de sus medias en cazadores-recolectores, JP-1, agricultores, RA-1.

Estos resultados permitieron considerar que los elevados tamaños dentales presentados por los individuos de CS-1 (Figura 8) 
a)

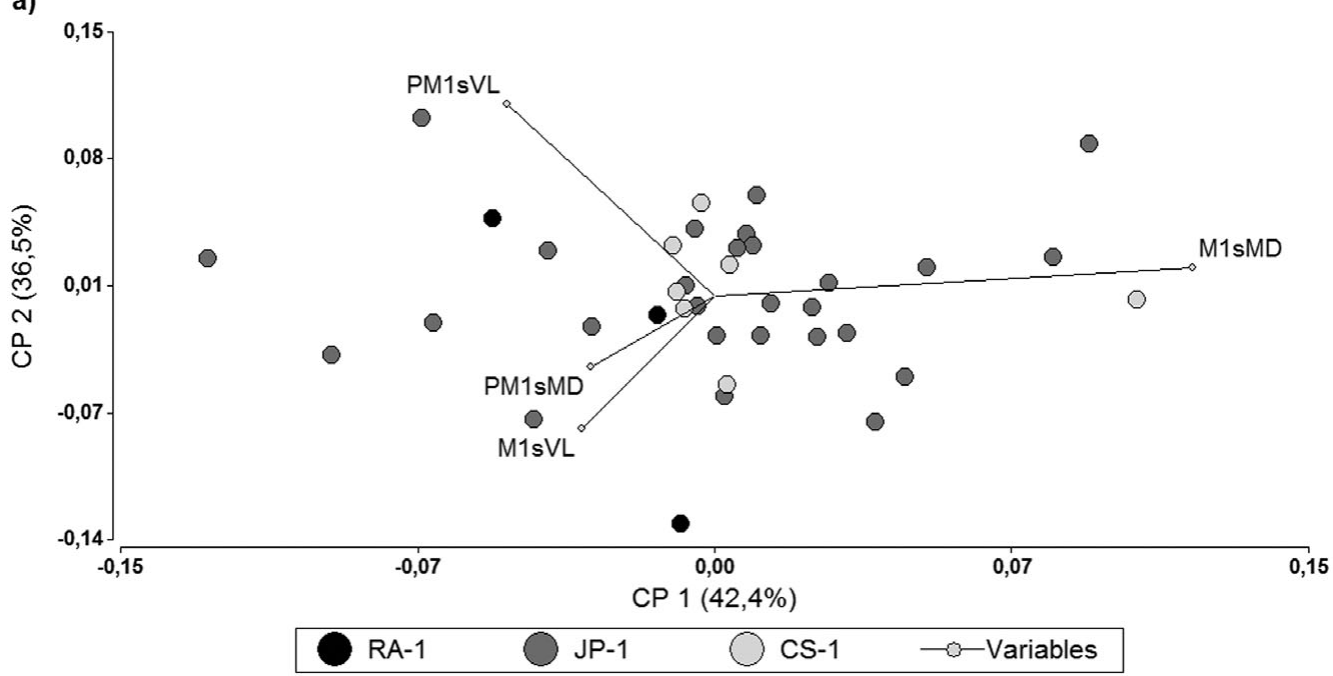

b)

RA-1

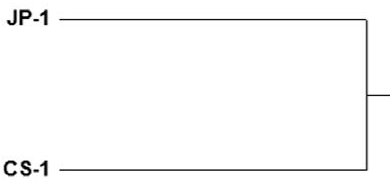

0,00

0,01

0,03

0,04

0,05

Figura 7. (a) Diagrama de dispersión biespacial del ACP para individuos de CS-1, JP-1 y RA-1. CP: Componente principal; PM: premolar; M: molar; s: superior; MD: diámetro mesio-distal; VL: diámetro vestíbulo lingual. (b) Dendrograma que describe el agrupamiento de JP-1, RA-1 y CS-1 en función de las variables de forma dental de sus individuos.

podrían enmascarar diferencias entre el resto de las muestras. A fin de evaluar tal hipótesis se procedió a repetir el ANOVA, excluyendo del mismo a los individuos de CS-1. Como resultado se obtuvo un $F=15,76$ y un valor de $p$ inferior a 0,0001 (Tabla Suplementaria 5), constatando la existencia de diferencias altamente significativas entre las muestras analizadas. Por su parte la prueba DGC permitió diferenciar dos agrupamientos, uno conformado por las muestras de cazadores-recolectores y JP-1, y el otro definido por la muestra de agricultores y RA-1.

\section{Asociación entre Variación Morfológica e Isotópica}

Los resultados de las regresiones espaciales indican que la dieta isotópica explica gran parte de los patrones morfométricos observados tanto en cráneo como en dientes. Los valores de $\delta^{13} \mathrm{C}_{\text {col }}$ y $\delta^{13} C_{\text {ap }}$ muestran una alta correlación con la forma del cráneo completo (84-98\%) y de la bóveda (92-99\%), a pesar de que esta última refleja variaciones como producto de la presencia de modificaciones culturales del cráneo (Tabla Suplementaria 6). Asimismo, la diversidad de valores de $\delta^{15} \mathrm{~N}$ se corresponde significativa- 


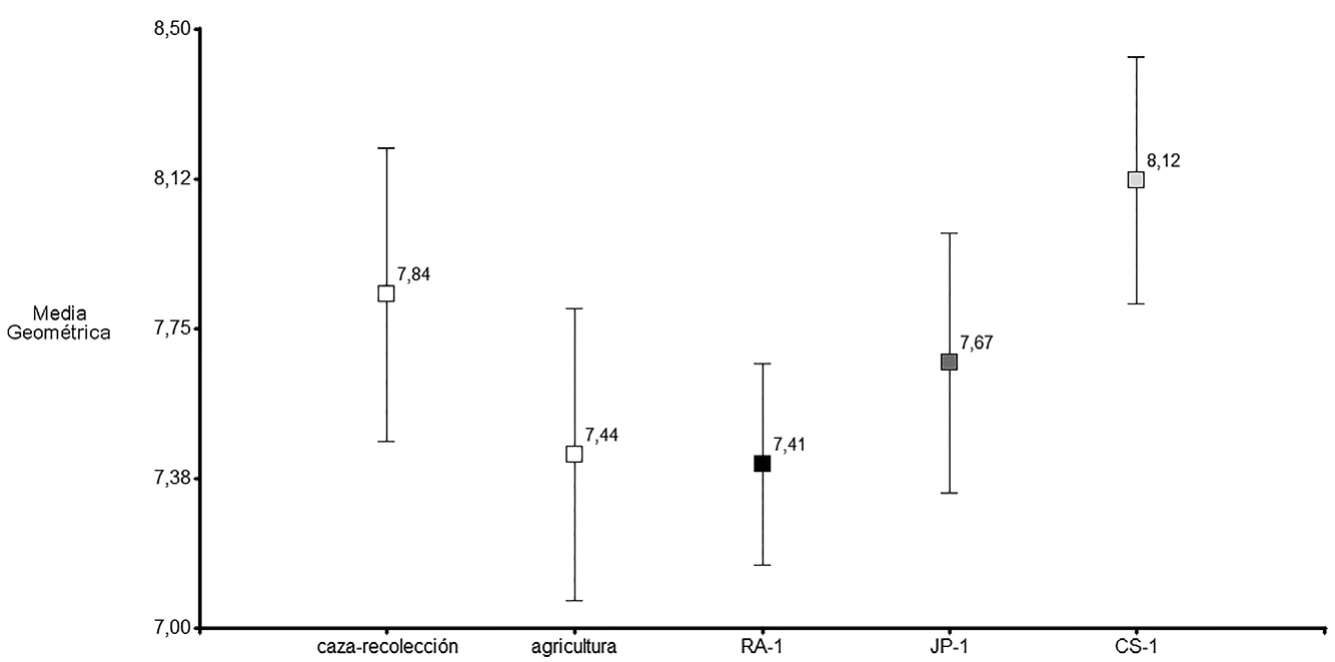

Figura 8. Variación del tamaño dental en JP-1, RA-1, CS-1 y grupos con estrategias de caza-recolección y agricultura.

mente con la variación del tamaño del cráneo completo (98\%), la mandíbula (78\%) y los dientes ( $82 \%)$, y con la forma de la base craneana (84\%), la mandíbula (84\%) y los dientes $(99 \%)$. Al contrario de lo esperado, el esqueleto facial, que es la estructura craneana que presenta mayor influencia del ambiente debido a su crecimiento más lento (Atchley y Hall 1991), no puede ser explicada por la diversidad isotópica. Sin embargo, tanto la variación en tamaño como de la forma de la mandíbula son explicados (entre un $78 \%$ y un $85 \%$ ) por los valores de $\delta^{15} \mathrm{~N}$, lo cual coincide con las expectativas. Finalmente, la forma de los dientes, que se espera varíe según procesos aleatorios (Hillson 1996), presenta una asociación alta con la diversidad en los valores de $\delta^{15} \mathrm{~N}$.

\section{Cultígenos y Variabilidad Humana en la Frontera Agrícola Prehispánica}

Estos resultados señalan para el sector límite de la agricultura tendencias heterogéneas y variables en la dieta, movilidad humana y morfometría craneofacial y dental. Dicha heterogeneidad habría tenido lugar durante el período que abarca los primeros ca. 500 años posteriores a la incorporación de plantas domésticas. Al comparar sitios cercanos geográficamente, se controla la variación ambiental, pudiendo enfocarse en la variabilidad espacial. Asimismo, la continuidad temporal de los conjuntos permite visualizar cambios ocurridos en un lapso de tiempo relativamente corto y cercano al momento de la aparición de cultígenos.

Las tendencias isotópicas en la fracción proteica mostraron que los valores de $\delta^{13} \mathrm{C}$ más elevados, que reflejan un mayor (aunque nunca elevado) consumo directo o indirecto de plantas $\mathrm{C}_{4}$, se encuentran en RA-1 mientras que los más negativos se observaron en JP-1. Por su parte, los valores de $\delta^{15} \mathrm{~N}$ más elevados, asociados a un incremento en el nivel trófico (importancia de carnes en la dieta, por ejemplo), se registran en CS-1 y luego JP-1. Los valores más bajos de esta relación isotópica se encuentran en RA-1, lo que implicaría para estos individuos dietas principalmente basadas en vegetales, aunque las diferencias no son significativas entre este sitio y JP-1. La aplicación de modelos bayesianos confirma estas tendencias tanto para la fracción proteica como para la dieta total (Figura Suplementaria 2). Los resultados coinciden con estudios previos respecto a la importancia del maíz (Bernal et al. 2016; Gil et al. 2010, 2011), a pesar de que Bernal y colaboradores (2016) proponen un alto consumo de vegetales entre las poblaciones del sur de 
Mendoza, lo que no se corrobora con los resultados aquí presentados para JP-1 y CS-1.

El estudio de la variación en la forma del cráneo completo, el esqueleto facial y las piezas dentales permitió diferenciar los individuos de RA-1 de los de CS-1 y JP-1. Estos últimos muestran mayores similitudes entre sí. Este patrón coincide con las diferencias observadas en las dietas inferidas para los tres sitios. Cuando se observa la variación en forma de la base craneana (Figura 6d) se nota otra tendencia, ubicándose en los extremos los individuos de JP-1 y CS-1 y quedando los de RA-1 con una posición intermedia. Esto a su vez coincide con la secuencia cronológica de los sitios.

La variación de tamaño en cráneo y mandíbula muestra un patrón común para ambas unidades anatómicas y coincidente con la tendencia en los isótopos $\delta^{13} \mathrm{C}$ y $\delta^{15} \mathrm{~N}$. Los individuos de CS-1 presentan las mayores dimensiones, seguidos por los de JP-1 y los de RA-1 con los tamaños más reducidos. Resulta significativo que dicho ordenamiento coincide con el que se obtiene al analizar los resultados del modelo de mezcla de dieta, que establece a CS-1 como el sitio con mayor consumo de recursos de alto nivel trófico (e.g., camélidos y dasipódidos) $y$ asocia a RA-1 con un mayor consumo de recursos vegetales (maíz y algarrobo), mientras que JP-1 presenta una disposición intermedia. El análisis comparativo de los tamaños dentales permitió establecer que CS-1 se diferencia significativamente del resto de las muestras consideradas, presentando los mayores tamaños. Por su parte, hay diferencias significativas entre los tamaños dentales de JP-1, los cuales se asocian a los de grupos cazadores-recolectores, y RA-1, que se agrupan con los de grupos agricultores. Dichos resultados son consistentes con análisis previos (Bernal et al. 2010; Sardi et al. 2006) que establecen relaciones entre la variación en tamaño de las estructuras biológicas (cráneo y las piezas dentales) y variaciones en factores relacionados a la dieta y los patrones de actividad producto de diferentes sistemas de subsistencia.

A partir de los resultados obtenidos se plantean dos escenarios posibles que expliquen la variación registrada y los resultados obtenidos para las regresiones espaciales. La primera alter- nativa considera que la variación morfológica registrada para los individuos de los tres sitios analizados es producto de dos conjuntos poblacionales con diferentes patrones de variación heredable. Las similitudes observadas entre JP-1 y CS-1 responderían a una relación evolutiva más estrecha entre estas poblaciones (posiblemente relaciones de ancestría común), y su diferenciación respecto de los individuos de RA-1 sería el resultado de la acción diferencial de procesos microevolutivos (e.g., deriva, flujo génico, selección natural) y los acontecimientos históricos que modelaron la diferenciación biológica de dichas poblaciones a nivel intra e inter-poblacional (e.g., expansión poblacional, extinción poblacional, efecto fundador). En este contexto la relación entre dieta (e.g., $\left.\delta^{15} \mathrm{~N}\right)$ y variación morfológica (e.g., base craneal y piezas dentales) evidenciada por las regresiones espaciales es explicada en función de una correlación entre los conjuntos poblacionales (JP-1/CS-1 y RA-1) y los sistemas de subsistencia empleados por los mismos (cazarecolección y agricultura, respectivamente), una tendencia similar a la notada en otras regiones límite de la agricultura (Bollongino et al. 2013). En este escenario la variación morfológica de los conjuntos poblacionales, y su diferenciación unos de otros, respondería a las historias evolutivas particulares de los mismos y los valores de la dieta asociados a tal variación serían el producto de modelos de subsistencia diferenciales empleados por dichos conjuntos. Apoyando esta consideración se destacan los patrones de entierro en estos sitios: tanto JP-1 como CS-1 son osarios con alto número de individuos, mientras que en RA-1 se registra un bajo número de individuos con igual predominio de entierros primarios y secundarios (Gil et al. 2008; Lagiglia 1991, 1994; Peralta 2015).

El segundo escenario establece que las diferencias en la dieta habrían determinado la diversificación de las poblaciones estudiadas, considerando que el cambio fenotípico persiste y se transmite lo suficientemente rápido a través de las generaciones como para alterar la trayectoria de un proceso ecológico que se encuentra aún en progreso. La existencia de dietas diferentes habría impactado sobre la plasticidad fenotípica de estas poblaciones durante el transcurso de las trayectorias ontogénicas de los individuos 
que la componen, dando lugar a la variabilidad morfológica observada. De esta manera RA-1, que presentaría morfologías y valores isotópicos esperables para grupos con una alta proporción de cultígenos en su dieta, se diferencia más claramente de JP-1 y CS-1, que a su vez se parecen entre sí, caracterizados por una proporción mayor de proteínas de distinto origen en su dieta.

Diversos trabajos han propuesto un incremento de la demografía humana en esta región alrededor de 2000 años aP, lo que habría impulsado un proceso de intensificación como una forma de adecuar la presión sobre los recursos (Gil et al. 2014b; Johnson et al. 2015; Neme 2007). En su primera formulación esta intensificación implicó un aumento en la amplitud de la dieta, disminución de la movilidad y la demarcación de territorios (Neme 2007). Como parte de esos cambios se habrían incorporado plantas domésticas en la dieta de las poblaciones. Aunque el proceso de intensificación no ha sido claramente registrado en el sur de Mendoza (Otaola 2012), las evidencias señalan el registro de macro-restos de plantas domésticas desde ca. 2000/2200 años aP y contemporáneamente un aumento demográfico inferido en la tendencia de frecuencias radiocarbónicas (Gil et al. 2014b). Las variaciones en la demografía humana, así como en la abundancia y variación de los recursos silvestres, explicarían la importancia que habrían tenido los recursos domésticos en estas poblaciones (Barlow 2002). Si bien se ha observado la incorporación de plantas domésticas a la dieta humana de la región hace ca. 2200 años cal aP, las mismas no habrían sido la base de la alimentación. La evidencia presentada en este trabajo sugiere que no existe una profundización temporalmente continua en la importancia de los cultígenos, ni tampoco señala un sistema de subsistencia estable durante el tiempo aquí comprendido. Por el contrario, se destaca un flujo de avances y retrocesos de estrategias o poblaciones (demes), caracterizados por pulsos donde dominan unas más orientadas a la explotación de recursos silvestres y otras más vinculadas con la explotación de recursos domésticos. Los cambios no fueron lineales en el tiempo ya que JP-1, el más antiguo de los sitios, se diferencia significativamente de RA-1, que es el sitio que cronológicamente lo continúa, mientras que se asemeja a CS-1, con el que tiene baja probabilidad de contemporaneidad. Estas disrupciones temporales señalarían un sistema en equilibrio oscilante manteniendo la importancia de los recursos domésticos por debajo de umbrales de no retorno (Freeman et al. 2015).

$\mathrm{Si}$ se asume que las dietas de los sitios JP-1 y CS-1 corresponden a poblaciones que basaron su subsistencia en recursos silvestres, especialmente animales, y que tendrían diferencias significativas en la proporción de plantas $\mathrm{C}_{4}$ consumidas en relación con los individuos de RA-1, entonces el enriquecimiento que muestran los valores de $\delta^{13} \mathrm{C}$ en los individuos de CS-1 respecto de JP-1 debería ser atribuido al mayor consumo de recursos animales con valores de $\delta^{13} \mathrm{C}$ enriquecidos como los dasipódidos. Como ya se mencionó, los altos valores de $\delta^{15} \mathrm{~N}$ que muestran los individuos de CS-1 refuerzan la idea de un alto consumo de recursos animales, y no así de vegetales, debilitando la posibilidad de un enriquecimiento causado por el consumo de plantas $\mathrm{C}_{4}$ como el maíz. Los resultados de los modelos de mezcla de dieta también sugieren un mayor consumo de animales con valores de $\delta^{13} \mathrm{C}$ y $\delta^{15} \mathrm{~N}$ enriquecido como los dasipódidos.

\section{Conclusión}

La arqueología del centro-occidente asumió que una vez establecidos los primeros grupos de agricultores, los mismos habrían continuado en la región profundizando su dependencia agrícola, incorporando la tecnología cerámica y disminuyendo la movilidad. Nuestros resultados basados en el registro de los tres sitios presentados indican que en el límite de la agricultura prehispánica la organización de los sistemas humanos, principalmente señalados por su dieta y su movilidad, fue altamente variable entre ca. 1900 y 1200 años cal aP. Esta variabilidad se asocia con diferenciaciones en la morfometría craneofacial y dental, que señalan patrones compatibles con poblaciones con un fuerte énfasis en la explotación de recursos silvestres (JP-1 y CS-1), intercalados con otros donde el consumo de alimentos domésticos pudo ser más significativo (RA-1). En ningún caso los estudios de isótopos señalan un fuerte énfasis en el maíz, aunque para 
RA-1 este podría haber sido más significativo que para el resto de los sitios analizados. Estos resultados no confirman una progresiva profundización en la articulación de recursos domésticos y sedentarismo. Por el contrario, señalan una oscilación en la importancia de los mismos, la cual se refleja en una alta variabilidad en la organización de los sistemas humanos, una vez que los recursos domésticos llegaron a la región. Esta oscilación en la organización de los sistemas pudo continuar hasta tiempos históricos, donde la información escrita señala ocupaciones de cazadores-recolectores en este valle, que 2.200 años atrás habría sido posiblemente el límite de grupos agricultores. Esta variabilidad propuesta en el trabajo es difícilmente detectada cuando el registro arqueológico es ordenado y articulado considerando unidades culturales definidas sobre la base de uno o varios rasgos diagnósticos. Dado el tamaño de la muestra creemos que este trabajo tiene un sentido exploratorio, señalando un patrón no detectado previamente con el potencial de ser corroborado o desestimado en futuras investigaciones.

Agradecimientos. Este trabajo se realizó en el marco de los proyectos PICT 2013-0881, PICT 2016-2667 y PIPCONICET 0342. Agradecemos al Laboratorio de Isótopos Estables en Ciencias Ambientales (LIECA). Tres evaluadores señalaron aspectos que ayudaron a mejorar sustancialmente nuestra primera versión de este trabajo. Agradecemos a Miguel Giardina por ayudarnos con la preparación de figuras y discutir algunas ideas. Al Museo de Historia Natural de San Rafael por permitir el estudio de estos materiales.

Declaración de Disponibilidad de Datos. Los datos físicos y digitales sobre los cuales se basa la investigación se encuentran disponibles en el Museo de Historia Natural de San Rafael. Para consultarlos, pueden contactarse con cualquiera de los autores.

Materiales Suplementarios. Para acceder a los materiales suplementarios que acompañan este artículo visitar https:// doi.org/10.1017/laq.2017.59

Figura Suplementaria 1. Rangos esperados para valores de $\delta^{18} \mathrm{O}$ en muestras óseas humanas según la distribución espacial de aguas y valores obtenidos.

Figura Suplementaria 2. Tendencia y variabilidad de la dieta según el colágeno (proteínas) y apatita (dieta total).

Tabla Suplementaria 1. Número Mínimo de Individuos por sitio y Tamaño de la Muestra para cada Variable Analizada.
Tabla Suplementaria 2. Tendencias en los Isótopos Estables $\left(\delta^{13} \mathrm{C}\right.$ y $\left.\delta^{15} \mathrm{~N}\right)$ en los Taxones Vegetales y Animales de la Región.

Tabla Suplementaria 3. Coeficientes de Ponderación de las Variables de Forma para CP1 y CP2.

Tabla Suplementaria 4. Resultados del ANOVA y Test DGC para Medias Geométricas de CS-1, JP-1, RA-1, y Grupos Cazadores-Recolectores y Agricultores.

Tabla Suplementaria 5. Resultados del ANOVA y Test DGC para Medias Geométricas de JP-1, RA-1, y Grupos Cazadores-Recolectores y Agricultores.

Tabla Suplementaria 6. Resultados de Regresiones Espaciales a partir de los Valores de $\mathrm{R}^{2}$.

Texto Suplementario 1 . Variación Isotópica $\left(\delta^{18} \mathrm{O}\right)$ espacial de las aguas superficiales.

Texto Suplementario 2. Metodología.

\section{Referencias Citadas}

Ambrose, Stanley H.

1990 Preparation and Characterization of Bone and Tooth Collagen for Isotopic Analysis. Journal of Archaeological Science 17:431-451.

Atchley, William R. y Brian K. Hall

1991 A Model for Development and Evolution of Complex Morphological Structures. Biological Reviews 66:101-157.

Barlow, K. R

2002 Predicting Maize Agriculture Among the Fremont: An Economic Comparison of Farming and Foraging in the American Southwest. American Antiquity 67: 65-87.

Bernal, Valeria

2008 Procesos de diferenciación biológica entre poblaciones humanas del Holoceno tardío de Patagonia. Una aproximación desde la variación métrica dental. Tesis doctoral inédita, Universidad Nacional de La Plata, La Plata, Buenos Aires, Argentina.

Bernal, Valeria, S. Iván Pérez, Paula N. González, Marina L. Sardi y Héctor M. Pucciarelli

2010 Spatial Patterns and Evolutionary Processes in Southern South America: A Study of Dental Morphometric Variation. American Journal of Physical Anthropology 142:95-104.

Bernal, Valeria, Paula N. González, Florencia Gordón y S. Iván Pérez

2016 Exploring Dietary Patterns in the Southernmost Limit of Prehispanic Agriculture in America by Using Bayesian Stable Isotope Mixing Models. Current Anthropology 57(2):230-239.

Bollongino, Ruth, Olaf Nehlich, Michael P. Richards, Jörg Orschiedt, Mark G. Thomas, Christian Sell, Zuzana Fajkošová, Adam Powell y Joachim Burger

20132000 Years of Parallel Societies in Stone Age Central Europe. Science 342:479-481.

Cohen, Mark N.

1977 The Food Crisis in Prehistory. Yale University Press, New Haven.

Coltrain, Joan y Steven Leavitt

2002 Climate and Diet in Fremont Prehistory: Economic Variability and Abandonment of Maize Agriculture in the Great Salt Lake Basin. American Antiquity 67:453485 . 
Dansgaard, Willi

1964 Stable Isotopes in Precipitation. Tellus 16(4):436468.

Dupras, Tosha y Henry P. Schwarcz

2001 Strangers in a Strange Land: Stable Isotope Evidence for Human Migration in the Dakhleh Oasis, Egypt. Journal of Archaeological Science 28(11):11991208.

Fernández, Fernando J.

2012 Microvertebrados del Holoceno de sitios arqueológicos en el sur de Mendoza (República Argentina): Aspectos tafonómicos y sus implicancias en la subsistencia humana. Tesis doctoral inédita. SEDICI Universidad Nacional de La Plata, La Plata, Buenos Aires, Argentina.

Freeman, Jacob, Matthew A. Peeples y John M. Anderies 2015 Toward a Theory of Non-linear Transitions from Foraging to Farming. Journal of Anthropological Archaeology 40:109-122.

Gat, Joel R.

1996 Oxygen and Hydrogen Isotopes in the Hydrologic Cycle. Annual Review of Earth and Planetary Sciences 24:225-262.

Giardina, Miguel A., Gustavo A. Neme y Adolfo F. Gil

2014 Rheidae Egg Human Exploitation and Stable Isotopes: Trends from West Central Argentina. International Journal of Osteoarchaeology 24:166-186.

Giardina, Miguel A., Mercedes Corbat, Eva Peralta, Gregoria Cochero, Fernando Franchetti y Laura Salgán

2015 El registro arqueológico en sitio La Olla (San Rafael, Mendoza): Implicaciones para las ocupaciones humanas en el valle medio del río Atuel. Revista del Museo de Antropología 8(1):51-66.

Gil, Adolfo F.

1998 Cultígenos prehispánicos en el Sur de Mendoza. Discusión en torno al límite meridional de la agricultura andina. Relaciones 22-23:295-318.

2003 Zea Mays on the South American Periphery: Chronology and Dietary Importance. Current Anthropology 44(2):295-300.

Gil, Adolfo F., Gustavo A. Neme, Alicia Hernández, Paula Novellino, Miguel A. Giardina, Laura Salgán, Hugo Tucker y Elina Albarrán

2008 Rincón del Atuel-1 (San Rafael, Mendoza): Evidencias arqueológicas e implicancias regionales. Intersecciones 9:113-132.

Gil, Adolfo F. y Gustavo A. Neme

2010 Registro arqueológico en la cuenca media del Atuel: Viejos y nuevos problemas; viejos y nuevos datos. En Condiciones paleoambientales y ocupaciones humanas durante la transición Pleistoceno-Holoceno y Holoceno de Mendoza, editado por Marcelo Zárate, Adolfo Gil y Gustavo Neme, pp. 239-275. Sociedad Argentina de Antropología, Buenos Aires.

2013 Fronteras en el registro arqueológico: Preguntas, casos y debates en el Centro Occidente Argentino. En Fronteras y periferias en arqueología e historia, editado por Margarita Gascón y María José Ots, pp. 11-29. Ediciones Dunken, Buenos Aires.

Gil, Adolfo F., Gustavo A. Neme y Robert H. Tykot

2010 Isótopos estables y consumo de maíz en el Centro Occidente Argentino: Tendencias temporales y espaciales. Chungara 42(2):497-513.

2011 Stable Isotopes and Human Diet in Central Western Argentina. Journal of Archaeological Science 38:13951404.
Gil, Adolfo F., Miguel A. Giardina, Gustavo A. Neme y Andrew Ugan

2014a Demografía humana e incorporación de cultígenos en el centro occidente argentino: Explorando tendencias en las fechas radiocarbónicas. Revista Española de Antropología Americana 44(2):523-553.

Gil, Adolfo F., Gustavo A. Neme, Andrew Ugan y Robert H. Tykot

2014b Oxygen Isotopes and Human Mobility in Central Western Argentina. International Journal of Osteoarchaeology 24:31-41.

Hillson, Simon

1996 Dental Anthropology. Cambridge University Press, Cambridge.

Hillson, Simon, Charles FitzGerald y Helen Flinn

2005 Alternative Dental Measurements: Proposals and Relationships with Other Measurements. American Journal of Physical Anthropology 126:413-426.

Johnson, Amber L., Adolfo F. Gil, Gustavo A. Neme y Jacob C. Freeman

2015 Hierarchical Method Using Ethnographic Data Sets to Guide Archaeological Research: Testing Models of Plant Intensification and Maize Use in Central Western Argentina. Journal of Anthropological Archaeology 38:52-58.

Lagiglia, Humberto A.

1982 El proceso de agriculturización del Sur de Cuyo: La Cultura del Atuel II. Actas del V Congreso Nacional de Arqueología Argentina 1:231-252. San Juan, San Juan, Argentina.

1991 Acerca de los hallazgos indígenas de Cañada Seca. Boletín del Instituto de Ciencias Naturales 22:3-4. San Rafael, Mendoza, Argentina.

1994 El contexto arqueológico del cementerio Jaime Prats y su fechado C-14. Actas del IX Congreso Nacional de Arqueología Argentina 2:111-112. San Rafael, Mendoza, Argentina.

2001 Los orígenes de la agricultura en la Argentina. En Historia Argentina Prehispánica, editado por Eduardo Berberián y Axel Nielsen, Vol. 1, pp. 41-81. Editorial Brujas, Córdoba, Córdoba, Argentina.

2002 Arqueología prehistórica del sur mendocino y sus relaciones con el centro-oeste Argentino. En Entre montañas y desiertos: Arqueología del sur de Mendoza, editado por Adolfo F. Gil y Gustavo A. Neme, pp. 43-64. Sociedad Argentina de Antropología, Buenos Aires.

Llano, Carina y Diego Andreoni

2012 Caracterización espacial y temporal en el uso de los recursos vegetales entre los grupos cazadoresrecolectores del sur mendocino durante el Holoceno. En Paleoecología humana en el sur de Mendoza: Perspectivas arqueológicas, editado por Gustavo A. Neme y Adolfo F. Gil, pp. 57-84. Sociedad Argentina de Antropología, Buenos Aires.

Menéndez, Lumila y Federico Lotto

2016 Estudio morfométrico de las modificaciones culturales del cráneo en el Centro-Oeste de Argentina. Comechingonia 20(1):143-173.

Moore, Jonathan W. y Brice X. Semmens

2008 Incorporating Uncertainty and Prior Information into Stable Isotope Mixing Models. Ecology Letters 11:470-480.

Neme, Gustavo A.

2007 Cazadores-recolectores de altura en los Andes meridionales: El alto valle del río Atuel. British 
Archaeological Reports International Series 1591. Archaeopress, Oxford.

Neme, Gustavo A. y Adolfo F. Gil

2008 Biogeografía humana en los Andes Meridionales: Tendencias arqueológicas en el Sur de Mendoza. Chungara 40:5-18.

Otaola, Clara

2012 Procesos de intensificación y aprovechamiento de médula y grasa ósea en el sur de Mendoza, Argentina. Archaeofauna 21:235-247.

Parnell, Andrew C., Donald L. Phillips, Stuart Bearhop, Brice X. Semmens, Eric J. Ward, Jonathan W. Moore, Andrew L. Jackson, Jonathan Grey, David J. Kelly y Richard Inger

2013 Bayesian Stable Isotope Mixing Models. Environmetrics 24:387-399.

Peralta, Eva A.

2015 Subsistencia y movilidad en la cuenca media del Atuel en el Holoceno Tardío: Una perspectiva bioarqueológica, aplicando el análisis de marcadores de estrés ocupacional. Tesis de licenciatura inédita, Facultad de Filosofía y Letras, Universidad de Buenos Aires, Buenos Aires.

Phillips, Donald L.

2012 Converting Isotope Values to Diet Composition: The Use of Mixing Models. Journal of Mammalogy 93(2):342-352.

Phillips, Donald L., Richard Inger, Stuart Bearhop, Andrew L. Jackson, Jonathan W. Moore, Andrew C. Parnell, Brice X. Semmens y Eric J. Ward

2014 Best Practices for Use of Stable Isotope Mixing Models in Foodweb Studies. Canadian Journal of Zoology 92:823-835.

Sardi, Marina L., Paula S. Novellino y Héctor M. Pucciarelli 2006 Craniofacial Morphology in the Argentine CenterWest: Consequences of the Transition to Food Production. American Journal of Physical Anthropology 130:333-343.

Schafer, Joseph L.

1999a Multiple Imputation: A Primer. Statistical Methods in Medical Research 8:3-15. 1999b NORM Users' Guide (Version 2). The Pennsylvania State University, University Park, Pennsylvania.

Scott, George R.

2008 Dental Morphology. En Biological Anthropology of the Human Skeleton, editado por M. Anne Katzenberg y Shelley R. Saunders, pp. 265-298. Wiley-Liss, New York.

Smith, Bruce

2001 The Transition to Food Production. En Archaeology at the Millennium. A Sourcebook, editado por Gary M. Feinman y T. Douglas Price, pp.199-229. Plenum Press, New York.

Stuiver, Minze, Paula J. Reimer y Ron W. Reimer

2017 CALIB 7.1. http://calib.org, accedido el 10 de agosto de 2017.

Szpak, Paul, Christine D. White, Fred J. Longstaffe, JeanFrancois Millaire y Víctor F. Vásquez Sánchez

2013 Carbon and Nitrogen Isotopic Survey of Northern Peruvian Plants: Baselines for Paleodietary and Paleoecological Studies. PLoS ONE 8(1): e53763. http://dx. doi.org/10.1371/journal.pone.0053763, accedido el 10 de agosto de 2017.

Sponheimer, Matt y Julia A. Lee-Thorp

1999 Oxygen Isotopes in Enamel Carbonate and Their Ecological Significance. Journal of Archaeological Science 26(6):723-728.

Stock, Brian C. y Brice X. Semmens

2013 MixSIAR GUI User Manual, Version 1.0. Documento electrónico, http://conserver.iugocafe.org/user/ brice.semmens/MixSIAR, accedido el 10 de abril 2017.

Ugan, Andrew, Gustavo A. Neme, Adolfo F. Gil, Joan Coltrain, Robert H. Tykot y Paula S. Novellino

2012 Geographic Variation in Bone Carbonate and Water $\delta 180$ Values in Mendoza, Argentina and Their Relationship to Prehistoric Economy and Settlement. Journal of Archaeological Science 39:2752-2763.

Submitted April 27, 2017; Revised August 16, 2017; Accepted September 21, 2017 